\title{
ON SYMPLECTIC DYNAMICS NEAR A HOMOCLINIC ORBIT TO 1-ELLIPTIC FIXED POINT
}

\author{
LEV LERMAN, ANNA MARKOVA
}

\begin{abstract}
We study the orbit behavior of a 4-dimensional smooth symplectic diffeomorphism $f$ near a homoclinic orbit $\Gamma$ to a 1-elliptic fixed point under some natural genericity assumptions. A 1-elliptic fixed point has two real eigenvalues outside the unit circle and two on the unit circle. Thus there is a smooth 2-dimensional center manifold $W^{c}$ where the restriction of the diffeomorphism has the elliptic fixed point supposed to be generic (no strong resonances and first Birkhoff coefficient is nonzero). Then the Moser theorem guarantees the existence of a positive measure set of KAM invariant curves. $W^{c}$ itself is a normally hyperbolic manifold in the whole phase space and due to Fenichel results in every point on $W^{c}$ having 1-dimensional stable and unstable smooth invariant curves smoothly foliating the related stable and unstable manifolds. In particular, each KAM invariant curve has stable and unstable smooth 2-dimensional invariant manifolds being Lagrangian ones. Stable and unstable manifolds of $W^{c}$ are 3-dimensional smooth manifolds which are assumed to be transverse along homoclinic orbit $\Gamma$. One of our theorems presents conditions under which each KAM invariant curve on $W^{c}$ in a sufficiently small neighborhood of $\Gamma$ has four transverse homoclinic orbits. Another result ensures that under some Moser genericity assumption for the restriction of $f$ on $W^{c}$ saddle periodic orbits in the resonance zone also have homoclinic orbits in the whole phase space, though its transversality or tangency cannot be verified directly. All this implies the complicated dynamics of the diffeomorphism and can serve as a criterion of its non-integrability.
\end{abstract}

\section{INTRODUCTION AND SET-UP}

Any tools that can help us to understand that if a given Hamiltonian system is integrable or non-integrable and therefore has a complicated orbit behavior are of the great importance. There are well known criteria based on the Melnikov method [33, 26, 29, 37, [10, but they are mainly applicable for systems being nearly integrable.

There exist other classes of criteria based on the study of the orbit behavior in definitely non-integrable systems: if we know that some structures in the phase space are met only in non-integrable systems, then we may take the existence of such a structure in the phase space of a system under consideration as a criterion of its non-integrability. Such criteria are most efficient if the structures mentioned can be rather easily identified. To this type of criterion one can refer those based on the existence of homoclinic orbits to the different types of invariant sets, the most popular are those related to homoclinic orbits to different types of equilibria, periodic orbits and invariant tori $26,12,28,30,23,24,25,4,8$. Surely, non-integrability criteria are not the unique goal

2010 Mathematics Subject Classification. Primary 37J10, 37J30, 37J45, 70H07.

Keywords. 1-elliptic fixed point, homoclinic, invariant curve, periodic orbits.

The authors thank R. de la Llave and S. V. Gonchenko for useful discussions.

The authors acknowledge partial support from the Russian Foundation for Basic Research under the grants 13-01-00589a (first author) and 14-01-00344 (second author).

The first author is also grateful for support from the Russian Ministry of Science and Education (project 1.1410.2014/K, target part) and from the Russian Science Foundation (project 14-41-00044). 
of the study: a much more interesting and harder problem is to describe possible types of orbit behavior in the system near such structure and its changes when parameters of the system vary.

In the paper we study a $C^{r}$-smooth, $r \geq 6$, symplectic diffeomorphism $f$ on a $C^{\infty}$ smooth 4-dimensional symplectic manifold $(M, \Omega) ; \Omega$ is a $C^{\infty}$-smooth non-degenerate 2 -form. We assume $f$ to have a 1-elliptic fixed point $p$; that is, differential $D f_{p}$ has one pair of multipliers $e^{ \pm i \alpha}$ on the unit circle and a pair of real multipliers $\mu, \mu^{-1}, \mu \neq \pm 1$. Below we suppose $\mu$ to be positive and $0<\mu<1$. We call such a fixed point an orientable 1-elliptic point. The fixed point with negative $\mu, \mu^{-1}$ we call non-orientable. The nonorientable point becomes orientable if one considers $f^{2}$ instead of $f$.

Near a 1-elliptic fixed point there is a $C^{r-1}$-smooth 2-dimensional invariant symplectic center submanifold $W^{c}$ corresponding to multipliers $e^{ \pm i \alpha}$ 22, 34. The restriction of $f$ on $W^{c}$ is a $C^{r-1}$-smooth 2-dimensional symplectic diffeomorphism and $p$ is its elliptic fixed point. We assume $p$ to be of the generic elliptic type [2] that is, strong resonances are absent in the system $(\alpha \neq \pi / 2,2 \pi / 3)$ and the first coefficient in the Birkhoff normal form for $\left.f\right|_{W^{c}}$ does not vanish. In this case we shall call a 1-elliptic fixed point a generic 1-elliptic fixed point. Then the Moser theorem [36] is valid for the restriction $\left.f\right|_{W^{c}}$ near $p$. This gives a positive measure Cantor set of closed invariant curves on $W^{c}$ which enclose $p$ and are accumulated to it. The needed minimal smoothness for a symplectic diffeomorphism is 5 due to 38 . This explains the inequality $r \geq 6$.

Center manifold $W^{c}$ is a normally hyperbolic invariant manifold in the sense of 15 , 21 and has its local $C^{r-1}$-smooth 3 -dimensional stable manifold $W_{\text {loc }}^{c s}$ and local $C^{r-1}$ smooth 3-dimensional unstable one $W_{\text {loc }}^{c u}$, since two other multipliers $\mu, \mu^{-1}$ are less than 1 and greater than 1, respectively (these two local 3-dimensional manifolds for the fixed point $p$ are simultaneously center-stable and center-unstable manifolds, respectively; this explains our notation). These manifolds can be extended till the global ones by the action of $f^{-1}$ and $f$, respectively. The extended manifolds will be denoted as $W^{c s}$ and $W^{c u}$.

Each invariant KAM-curve $\gamma$ on $W^{c}$ can be considered as being saddle one, since it has local 2-dimensional stable and unstable manifolds which can also be extended till global manifolds $W^{s}(\gamma), W^{u}(\gamma)$ by the action of $f, f^{-1}$. Topologically these manifolds are local cylinders, both being Lagrangian submanifolds in $M$ 1]. The existence and smoothness of these manifolds rely on the results of [14, 15] and will be proved in the Appendix.

Fixed point $p$ has also two $C^{r}$-smooth local invariant curves through $p$ being its local stable $W_{\mathrm{loc}}^{s}(p)$ and unstable $W_{\mathrm{loc}}^{u}(p)$ manifolds 22 . Their extensions by the action of $f^{-1}$ and $f$ are $C^{r}$-smooth invariant curves $W^{s}(p)$ and $W^{u}(p)$, respectively.

Our first two assumptions in the paper concern the existence of a homoclinic orbit to $p$ and its type.

Assumption 1 (Homoclinic intersection). Curves $W^{u}(p)$ and $W^{s}(p)$ have an intersection at some point $q$, thus generating a homoclinic orbit $\Gamma$ to fixed point $p$.

Assumption 2 (Transversality condition). Manifolds $W^{s}(p)$ and $W^{c u}(p)$ are transverse at point $q$ and, hence, along $\Gamma$.

Later in Section 3 we will construct linear symplectic scattering map $S$ which acts on tangent plane $T_{p} W^{c}$ and describes in the linear approximation an asymptotic behavior of orbits close to $\Gamma$ after one-round travel near $\Gamma$. The restriction of differential $D f_{p}$ on symplectic invariant plane $T_{p} W^{c} \subset T_{p} M$ is a linear symplectic 2-dimensional map with two eigenvalues $e^{ \pm i \alpha}$, and, therefore, this plane is foliated into closed invariant curves of the map. Every such curve is an ellipse, all of which can be obtained from the one multiplying their vectors at positive constants. Fix one such ellipse $E \subset T_{p} W^{c}$. Then its image $S(E)$ is also an ellipse (usually not from the foliation) with the same center at 
the origin and of the same area with respect to the restriction of 2 -form $\Omega$ on this plane. Thus, the intersection $E \cap S(E)$ either consists of four points (a generic case) or these two ellipses coincide (a degenerate case). In the first case the intersection of two ellipses is transverse at every four points.

Assumption 3 (Genericity condition). The intersection $E \cap S(E)$ is transverse and therefore consists of four points.

It is evident that this assumption does not depend on the explicit choice of the ellipse $E$. This condition allows one to select a generic case and provides the mean to verify this.

Our first result is the following theorem.

Theorem 1.1. Let a 4-dimensional symplectic diffeomorphism $f$ with 1-elliptic fixed point p obey Assumptions 1, 2, 3. Then there is a sufficiently small neighborhood $U$ of homoclinic orbit $\Gamma$ such that every closed invariant KAM-curve on $W^{c}(p) \cap U$ possesses four transverse homoclinic orbits in $U$.

Intersection of invariant manifolds of the diffeomorphism $f$ in the neighborhood of homoclinic orbits are sketchy, represented on Figure1 It is worth remarking that for our case center manifold $W^{c}$, as was mentioned, is normally a hyperbolic 2-dimensional invariant manifold on which the restriction of $f$ is a twist map. Thus our results on existence of transverse homoclinic orbits to invariant KAM-curves are connected with the study of Hamiltonian dynamics near low-dimensional invariant whiskered tori initiated in [13. and extended in many recent papers (see, for instance, reviews [27, 8, 11]).

Before going to the proof, let us recall some related results for Hamiltonian vector fields [30, 23, 24, 32, 18, 19, 35. Homoclinic orbits to a saddle-center equilibrium for a real analytic Hamiltonian system with two degrees of freedom, namely, for the restricted circular three body problem, were found numerically in 31] and proved to exist analytically through asymptotic expansions in 32 . The problem on the orbit behavior of a real analytic Hamiltonian system near a homoclinic orbit to a saddle-center equilibrium was first set up and partially solved in 30, though it was earlier discussed in [7. In particular, the existence of four transverse homoclinic orbits to every small (Lyapunov) periodic orbit on the center manifold of the saddle-center was proved in 30. using the Moser normal form, and the needed genericity condition was found first in [30. In [18, under an additional assumption that a homoclinic orbit to a saddle-center belongs to some invariant symplectic 2-dimensional submanifold (that is generically not the case), the genericity condition was reformulated in terms of the related scattering problem for the transverse 2-dimensional system linearized at the homoclinic orbit. It was first discovered in 35 and in a more refined invariant form in 19 that in a generic 1-parameter unfolding of reversible two Hamiltonian systems that unfold a Hamiltonian system with a symmetric homoclinic orbit to a symmetric saddle-center equilibrium, there exists a (self-accumulated) countable set of parameter values near the critical one such that for a point of this set the related Hamiltonian system has a homoclinic orbit to its symmetric saddle-center. Usually these latter orbits are multi-round with respect to the initial homoclinic orbit. Several applications where non-integrability of a system under consideration was proved using this method can be found in 6, 20. A partial extension of results to the case of Hamiltonian systems with $n$ degrees of freedom, $n \geq 3$, having a center-saddle equilibrium (one pair of pure imaginary eigenvalues and the remaining ones with non-zero real parts) with a homoclinic orbit was given in 24. Here the scattering map was extended onto the case when the center manifold is 2-dimensional but the dimension of transverse directions is $2(n-1)$. 


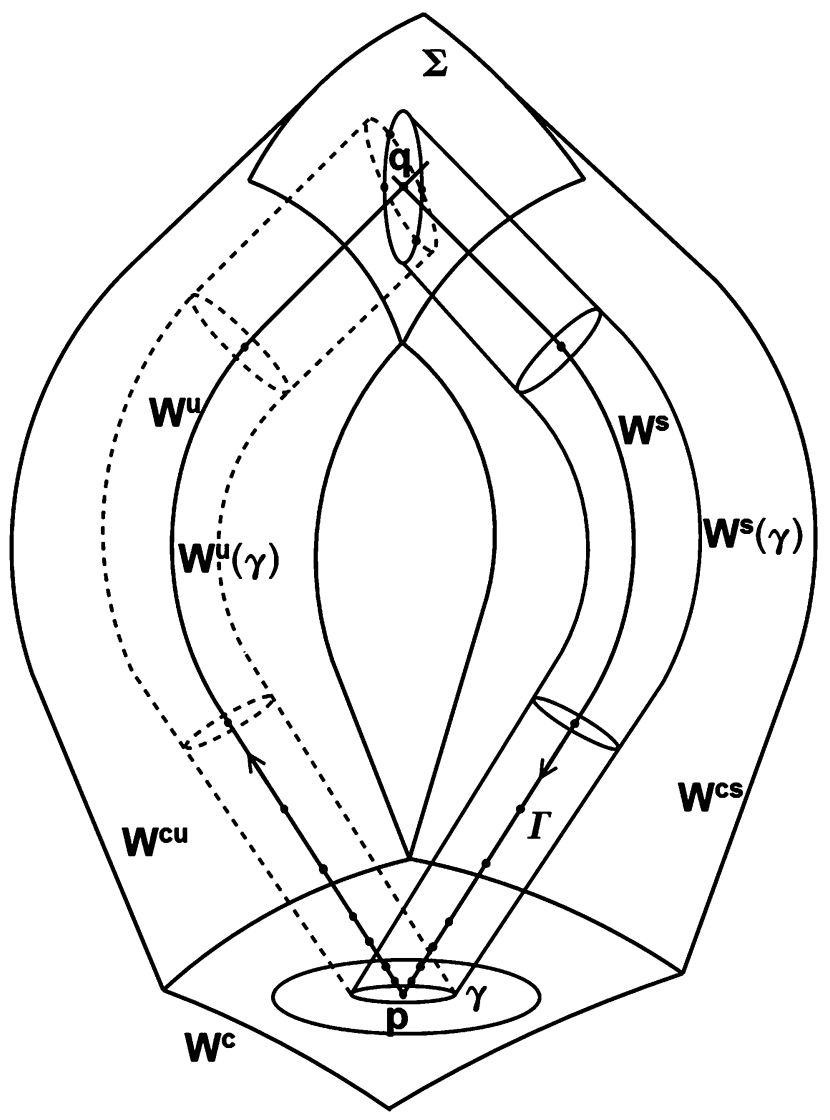

FigURE 1. Invariant manifolds in the neighborhood of homoclinic orbit

In fact, the results we discuss here refer to a 3 d. o.f. Hamiltonian system on a $C^{\infty}$ smooth symplectic manifold with a smooth Hamiltonian $H$ such that $X_{H}$ has a periodic orbit $\mathcal{C}$ of the center-saddle type. The latter means the multipliers of this orbit (except for the common double unit) are a pair $e^{ \pm i \alpha}$ and a pair of reals $\mu, \mu^{-1}, \mu \neq \pm 1$. Such periodic orbit has 2-dimensional stable and unstable invariant manifolds through $\mathcal{C}$, and they both belong to 5-dimensional level $H=H(\mathcal{C})$. If these manifolds have an intersection along some orbit $\Gamma$, then this homoclinic orbit tends to $\mathcal{C}$ as $t \rightarrow \pm \infty$. Choose some cross-section $N$ to the flow through a point $p \in \mathcal{C}$ in 5-dimensional level $H=H(\mathcal{C})$. We get a 4-dimensional symplectic (w.r.t. the restriction of 2-form $\Omega$ to $N$ ) local Poincaré diffeomorphism $f: N \rightarrow N$ with fixed point $p$ of the 1-elliptic type (corresponding to $\mathcal{C}$ ) defined in a neighborhood $U \subset N$ of $p$. Intersection of stable and unstable manifolds of $\mathcal{C}$ with $N$ give smooth local curves through the fixed point, and the traces of $\Gamma$ in $N$ form a countable set of homoclinic points accumulating at $p$. Fix one homoclinic point $q_{-} \in U$ on the unstable curve and one homoclinic point $q_{+} \in U$ on the stable curve. Choose some small neighborhoods $V_{-} \subset U$ of $q_{-}$and $V_{+} \subset U$ of $q_{+}$on $N$. Flow orbits define a symplectic map $F: V_{-} \rightarrow V_{+}, F\left(q_{-}\right)=q_{+}$, which we call a global one. Then a symplectic first return map defined as $f$ for points which belong to $U \backslash V_{-}$and as $F$ for points in $V_{-}$ is a map we discuss.

The local center manifold $W^{c}(\mathcal{C})$ for periodic orbit $\mathcal{C}$ is of dimension four, it contains the symplectic cylinder filled with periodic orbits (continuations of $\mathcal{C}$ onto close 
levels of $H$ ) and if conditions of Theorem 1.1 hold, then the restriction of the system on $W^{c}(\mathcal{C})$ has a positive measure set of invariant 2-dimensional tori with Diophantine rotation numbers. When we fix the level $H=H(\mathcal{C})$, its intersection with the center manifold is 3-dimensional. Every torus $\mathcal{T}$ has stable and unstable 3-dimensional manifolds which intersect each other along four transverse homoclinic orbits to the torus within 5-dimensional level $H=H(\mathcal{C})$.

\section{Consequences of the transversality Condition}

Due to Assumptions 1 1 and 2, two smooth 3-dimensional manifolds $W^{c s}(p)$ and $W^{c u}(p)$ intersect transversally at a homoclinic point $q$ and thus along a smooth 2-dimensional disk $\Sigma$ containing $q$. This disk is symplectic w.r.t. 2 -form $\omega$ being the restriction of 2 -form $\Omega$ on $\Sigma$. Indeed, in Section 4 it will be proved that in normalized coordinates in which $\Omega=d x \wedge d y+d u \wedge d v$ the disk (more exactly, some its finite iteration under $f$ ) will have the following representation:

$$
x=\Phi(u, v), \quad y=0 .
$$

This implies that $\Sigma$ is symplectic w.r.t. 2 -form $\omega=d u \wedge d v$. The following lemma is valid:

Lemma 2.1. If Assumptions 1 and 2 hold, then $\Sigma$ and $W^{u}(p)$ are transverse at $q$ within $W^{c u}(p)$ and, therefore, $W^{u}(p)$ and $W^{c s}(p)$ are also transverse at $q$.

Proof. To prove this lemma, we use some symplectic coordinates $(x, u, y, v), \Omega=d x \wedge d y$ $+d u \wedge d v$, in a neighborhood $V$ of point $p$ in which manifolds $W^{c u}$ and $W^{c s}$ are straightened; that is, they are given as $x=0$ (for $W^{c u}$ ) and $y=0$ (for $W^{c s}$ ). In addition, in these coordinates local stable manifold $W^{s}(p)$ is given as $y=u=v=0$, and local unstable manifold $W^{u}(p)$ is done as $x=u=v=0$. The existence of such coordinates is proved in Appendix 7.1. We also assume that $q \in V$. Since orbit $\Gamma$ through $q$ is homoclinic, there is an integer $N>0$ such that $f^{-n}(q) \in V$ for all $n \geq N$. Denote by $q_{1}$ the point $f^{-N}(q) \in V$ and let $l^{u}$ be the tangent to $W^{u}(p)$ at $q_{1}$. Denote $L=D f_{q_{1}}^{N}: T_{q_{1}} M \rightarrow T_{q} M$; then $L\left(l^{u}\right)$ is transversal to $T_{q} W^{c s}$ by virtue of Assumption 2 (transversality condition). Set $D=L\left(T_{q_{1}} W^{c u}\right) \cap T_{q} W^{c s} ; D$ is a 2 -dimensional plane. One needs to prove that $l^{s}$ (the tangent to $W^{s}(p)$ at $q$ ) does not belong to $D$, that is, intersects $D$ at only one point. For linear symplectic map $L$ the following matrix representation holds:

$$
L=\left(\begin{array}{ll}
a & b \\
c & d
\end{array}\right)=\left(\begin{array}{llll}
a_{11} & a_{12} & b_{11} & b_{12} \\
a_{21} & a_{22} & b_{21} & b_{22} \\
c_{11} & c_{12} & d_{11} & d_{12} \\
c_{21} & c_{22} & d_{21} & d_{22}
\end{array}\right)
$$

where $a, b, c, d$ are $2 \times 2$-matrices. Since $W^{c u}, W^{c s}$ are straightened in coordinates we use, tangent spaces to $W^{c u}, W^{u}(p)$ at $q_{1}$ and tangent spaces to $W^{c s}, W^{s}(p)$ at $q$ are written as follows:

$$
T_{q_{1}} W^{c u}=\left(\begin{array}{c}
0 \\
u \\
y \\
v
\end{array}\right), \quad T_{q} W^{c s}=\left(\begin{array}{c}
\bar{x} \\
\bar{u} \\
0 \\
\bar{v}
\end{array}\right), \quad l^{u}=\left(\begin{array}{c}
0 \\
0 \\
y \\
0
\end{array}\right), \quad l^{s}=\left(\begin{array}{c}
\bar{x} \\
0 \\
0 \\
0
\end{array}\right) .
$$

Transversality of $L\left(l^{u}\right)$ and $T_{q} W^{c s}$ is expressed as $d_{11} \neq 0$ in matrix $L$. Indeed, one has $L\left(l^{u}\right)=\left(b_{11} y, b_{21} y, d_{11} y, d_{21} y\right)^{T}$ (vector-column). Transversality of $L\left(l^{u}\right)$ and $T_{q} W^{c s}$ 
means that determinant

$$
\left|\begin{array}{llll}
b_{11} & 1 & 0 & 0 \\
b_{21} & 0 & 1 & 0 \\
d_{11} & 0 & 0 & 0 \\
d_{21} & 0 & 0 & 1
\end{array}\right|=d_{11}
$$

does not vanish.

The plane $D$ is given by the set of solutions of the system (1):

$$
D:\left\{\begin{array}{l}
\bar{x}=a_{12} u+b_{11} y+b_{12} v, \\
\bar{u}=a_{22} u+b_{21} y+b_{22} v, \\
0=c_{12} u+d_{11} y+d_{12} v, \\
\bar{v}=c_{22} u+d_{21} y+d_{22} v .
\end{array}\right.
$$

If $l^{s} \subset D$, then in the system above $\bar{u}=0, \bar{v}=0$ for all $(u, v)$. Expressing $y$ from the third equation in (11) and inserting into other equations we get a parametric representation of plane $D$ (with parameters $u, v$ ). Consider separately subsystem (2):

$$
\left\{\begin{array}{l}
a_{22} u+b_{21} y+b_{22} v=\bar{u} \\
c_{22} u+d_{21} y+d_{22} v=\bar{v}
\end{array}\right.
$$

Due to inequality $d_{11} \neq 0$ we can express $y=\left(-c_{12} u-d_{12} v\right) / d_{11}$ from (11) and insert it into (2):

$$
\left\{\begin{array}{l}
d_{11} a_{22} u+b_{21}\left(-c_{12} u-d_{12} v\right)+d_{11} b_{22} v=\bar{u} \\
d_{11} c_{22} u+d_{21}\left(-c_{12} u-d_{12} v\right)+d_{11} d_{22} v=\bar{v}
\end{array}\right.
$$

Let us calculate the determinant of the system (3). To this end, we rewrite it in the following form:

$$
\left\{\begin{array}{l}
u\left(d_{11} a_{22}-b_{21} c_{12}\right)+v\left(b_{22} d_{11}-b_{21} d_{12}\right)=\bar{u} \\
u\left(d_{11} c_{22}-d_{21} c_{12}\right)+v\left(d_{22} d_{11}-d_{21} d_{12}\right)=\bar{v}
\end{array}\right.
$$

This determinant is calculated as follows:

$$
\begin{aligned}
\Delta= & \left(d_{11} a_{22}-b_{21} c_{12}\right)\left(d_{22} d_{11}-d_{21} d_{12}\right)-\left(b_{22} d_{11}-b_{21} d_{12}\right)\left(d_{11} c_{22}-d_{21} c_{12}\right) \\
= & d_{11}^{2} a_{22} d_{22}-d_{11} a_{22} d_{21} d_{12}-b_{21} c_{12} d_{22} d_{11}+b_{21} c_{12} d_{21} d_{12}-d_{11}^{2} c_{22} b_{22} \\
& \quad+b_{22} d_{11} d_{21} c_{12}+b_{21} d_{12} d_{11} c_{22}-b_{21} d_{12} c_{12} d_{21} \\
= & d_{11}^{2} a_{22} d_{22}-d_{11} a_{22} d_{21} d_{12}-b_{21} c_{12} d_{22} d_{11} \\
& \quad-d_{11}^{2} c_{22} b_{22}+b_{22} d_{11} d_{21} c_{12}+b_{21} d_{12} d_{11} c_{22} \\
= & d_{11}^{2}\left(a_{22} d_{22}-c_{22} b_{22}\right)+d_{11} c_{12}\left(b_{22} d_{21}-b_{21} d_{22}\right)+d_{12} d_{11}\left(b_{21} c_{22}-a_{22} d_{21}\right) .
\end{aligned}
$$

Matrix $L$ is symplectic; therefore the following identities hold (see, for instance, [17]):

$$
a^{T} c \equiv c^{T} a, \quad b^{T} d \equiv d^{T} b, \quad d^{T} a-b^{T} c \equiv E=\left(\begin{array}{ll}
1 & 0 \\
0 & 1
\end{array}\right) .
$$

The first identity is equivalent to the equality

$$
a_{12} c_{11}+a_{22} c_{21}=c_{12} a_{11}+c_{22} a_{21} .
$$

Similarly, the second matrix identity is reduced to the equality

$$
b_{12} d_{11}+b_{22} d_{21}=d_{12} b_{11}+d_{22} b_{21} .
$$


The third matrix identity gives the following relations:

$$
\begin{aligned}
& d_{11} a_{11}+d_{21} a_{21}-b_{11} c_{11}-b_{21} c_{21}=1 \\
& d_{11} a_{12}+d_{21} a_{22}-b_{11} c_{12}-b_{21} c_{22}=0 \\
& d_{12} a_{11}+d_{22} a_{21}-b_{12} c_{11}-b_{22} c_{21}=0 \\
& d_{12} a_{12}+d_{22} a_{22}-b_{12} c_{12}-b_{22} c_{22}=1
\end{aligned}
$$

Now, taking into account relations (44) and the second and fourth equalities in (5), the expression for $\Delta$ can be transformed as follows:

$$
\begin{aligned}
\Delta=d_{11}^{2}\left(1+b_{12} c_{12}-d_{12} a_{12}\right)+d_{11} c_{12}\left(b_{11} d_{12}-\right. & \left.b_{12} d_{11}\right)+d_{12} d_{11}\left(d_{11} a_{12}-b_{11} c_{12}\right) \\
=d_{11}^{2}+d_{11}^{2} b_{12} c_{12}-d_{11}^{2} d_{12} a_{12} & +d_{11} c_{12} b_{11} d_{12}-d_{11}^{2} c_{12} b_{12} \\
& +d_{11}^{2} d_{12} a_{12}-d_{12} d_{11} b_{11} c_{12}=d_{11}^{2} \neq 0 .
\end{aligned}
$$

Thus, linear system (3) has a unique solution $(u, v)$ at the given $(\bar{u}, \bar{v})$. So, $\bar{u}=0, \bar{v}=0$ only if $(u, v)=(0,0)$ and $l^{s}$ intersects $D$ at the unique point.

Assumption 1 says that $f$ is degenerate since generically two smooth curves in a 4dimensional manifold do not intersect. This assumption selects a codimension 2 set of diffeomorphisms in the space of all $C^{r}$-smooth symplectic diffeomorphisms on $M$. Indeed, when a diffeomorphism with a homoclinic orbit to a 1-elliptic fixed point is perturbed within the class of smooth symplectic ones, for a perturbed $f^{\prime}$ the fixed point $p^{\prime}$ persists and its type is preserved. Therefore, due to the transversality condition, the intersection of perturbed $W^{c u}\left(p^{\prime}\right)$ and $W^{s}\left(p^{\prime}\right)$ persists as well, but the intersection point does not give generically a homoclinic orbit to $p^{\prime}$ : with backward iterations of $f^{\prime}$ the orbit through the intersection point can be either a heteroclinic orbit connecting $p^{\prime}$ and some invariant curve on $W^{c}\left(p^{\prime}\right)$ or some other orbit wandering near $W^{c}$. (Recall that if there are instability regions on $W^{c}\left(p^{\prime}\right)$, the orbit returns to $W^{c}\left(p^{\prime}\right)$ staying within 3-dimensional $W^{c u}\left(p^{\prime}\right)$. Thus it is locked between unstable 2-dimensional manifolds of invariant curves on $W^{c}\left(p^{\prime}\right)$, since they locally divide $W^{c u}\left(p^{\prime}\right)$.)

Nevertheless, if we turn to the related 3 d. o.f. Hamiltonian system with a periodic orbit $\mathcal{C}$ of 1-elliptic type (or it can be called of the saddle-center type), then such an orbit belongs to a smooth symplectic cylinder of periodic orbits of the same type. So, if $\mathcal{C}$ has a homoclinic orbit, then for the related close levels of Hamiltonian on the crosssection to $\mathcal{C}$ one gets a one-parameter family of symplectic Poincaré maps. Thus, if the Hamiltonian itself depends on a parameter $\varepsilon$ in a generic way, then the first return map for $\mathcal{C}$, derived by homoclinic orbits to it, unfolds to a two-parameter family of symplectic maps, and hence any close smooth one-parameter family of smooth Hamiltonians also has a 1-elliptic periodic orbit with homoclinic orbits to it. Thus, this phenomenon is generic for generic one-parameter unifoldings of a Hamiltonian with such structure.

Now we return to the problem under study. In a neighborhood of homoclinic point $q$ let us consider 2-dimensional symplectic disk $\Sigma$ through $q$ being the transverse intersection of extended 3-dimensional center-unstable manifold $W^{c u}(p)$ with 3-dimensional centerstable manifold $W^{c s}(p)$. Below we shall prove the existence of smooth stable and unstable manifolds for any KAM-curve on $W^{c}$ lying in a sufficiently small neighborhood of $p$. All stable manifolds belong to $W^{c s}$, and all unstable manifolds belong to $W^{c u}$. Hence, they intersect with $\Sigma$. The first statement concerning this intersection is the following:

Lemma 2.2. Disk $\Sigma$ contains two Cantor sets of smooth closed curves $w_{u}(\gamma)$ and $w_{s}(\gamma)$ being, respectively, traces of the related stable $W^{s}(\gamma)$ and unstable $W^{u}(\gamma)$ manifolds of invariant KAM-curves $\gamma \in W^{c}$. For fixed invariant curve $\gamma$ integrals of 2-form $\omega$ over 
disks $D^{u}(\gamma)$ and $D^{s}(\gamma)$ bounded by $w_{u}(\gamma)$ and $w_{s}(\gamma)$ are equal:

$$
\int_{D^{u}(\gamma)} \omega=\int_{D^{s}(\gamma)} \omega .
$$

Proof. The existence of stable manifold $W^{s}(\gamma)$ and unstable manifold $W^{u}(\gamma)$ of invariant KAM-curves $\gamma \in W^{c}$ will be proved in the Appendix.

The transversality condition implies the intersection of $W^{c u}$ with $W^{c s}$ near $q$ to occur along a smooth 2-dimensional disk $\Sigma$. For every invariant curve $\gamma$ in $W^{c}$ its stable manifold being extended by $f^{-1}$ in a finite number of iterations reaches a neighborhood of $q$ and transversely intersects $\Sigma$ within $W^{c s}(\gamma)$ along closed curve $w_{s}(\gamma)$; the trace of $W^{s}(p)$ is point $q$ itself. Traces on $\Sigma$ of $W^{u}(p)$ and $W^{u}(\gamma)$ in $W^{c u}(p)$ are respectively point $q$ and curve $w_{u}(\gamma)$.

Consider now a piecewise smooth 2-dimensional surface made up of a piece of the lateral side of the cylinder $W^{s}(\gamma)$ between $W^{c}$ and $\Sigma$, the piece of $W^{c}$ bounded by $\gamma$ and disk $\Sigma$. Integration of the form $\Omega$ over this surface is reduced to the difference of integrals over the disk in $W^{c}$ and that over disk in $\Sigma$ bounded by $w_{s}(\gamma)$, since the integral over the lateral side is equal to zero (it is a Lagrangian submanifold). This gives the equality of integrals in the statement of the lemma. Similarly, we get equality of the integral over the disk in $W^{c}$, bounded by $\gamma$, and the integral over disk in $\Sigma$, bounded by $w_{u}(\gamma)$.

\section{LiNEARIZATION AND SCATTERING MAP}

The genericity Assumption 3 is formulated using scattering map $S$. In this section we will construct this map, which acts on tangent plane $T_{p} W^{c}$. A scattering map is an analog of the scattering matrix for a Schrödinger type equation [42]. For the problems of the homoclinic dynamics related with non-hyperbolic equilibria, this map was first introduced in [23. A far-reaching extension of this map for a normally hyperbolic manifold in a Hamiltonian system was obtained in [11].

Consider first the linearization of the family of diffeomorphisms $f^{n}$ at homoclinic orbit

$$
\Gamma=\left\{q_{n}, n \in \mathbb{N} \mid q_{n+1}=f\left(q_{n}\right), q_{0}=q\right\} .
$$

This linearization is a sequence of linear symplectic maps

$$
L_{n}=\left.D f\right|_{T_{q_{n}} M}: T_{q_{n}} M \rightarrow T_{q_{n+1}} M
$$

and hence $\lim L_{n}=D f_{p}$ as $|n| \rightarrow \infty$. Since $f^{n}(q) \rightarrow p$ as $n \rightarrow \pm \infty$, there exists an integer $N$ large enough such that given a neighborhood $V$ of $p$ one gets $f^{n}(q) \in V$ for all $|n| \geq N$.

In neighborhood $V$ we choose a symplectic chart where fixed point $p$ is the origin; then map $f$ is in the standard form "linear diffeomorphism plus higher order terms". After a linear symplectic change of variables the linear part of the map can be transformed to the block-diagonal form:

$$
\left\{\begin{aligned}
x_{1} & =\mu x+\ldots, \\
y_{1} & =\mu^{-1} y+\ldots, \\
\left(\begin{array}{l}
u_{1} \\
v_{1}
\end{array}\right) & =\left(\begin{array}{rr}
\cos \alpha & -\sin \alpha \\
\sin \alpha & \cos \alpha
\end{array}\right)\left(\begin{array}{l}
u \\
v
\end{array}\right)+\ldots,
\end{aligned}\right.
$$

with $0<\mu<1$; dots mean terms of the order 2 and higher. In these coordinates the linearization of this discrete dynamical system at the homoclinic orbit $\Gamma$ is given as follows:

$$
\left\{\begin{array}{l}
\xi_{n+1}=\mu \xi_{n}+P_{n} \zeta_{n}, \\
\eta_{n+1}=\mu^{-1} \eta_{n}+Q_{n} \zeta_{n}, \\
\chi_{n+1}=R_{\alpha} \chi_{n}+W_{n} \zeta_{n},
\end{array}\right.
$$


where $\zeta_{n}=\left(\xi_{n}, \eta_{n}, \chi_{n}\right)^{\top}=\left(\xi_{n}, \eta_{n}, \chi_{n}^{1}, \chi_{n}^{2}\right)^{\top}$ is a coordinate 4-column vector in the tangent space at the point $q_{n}=\left(x_{n}, y_{n}, u_{n}, v_{n}\right) ; R_{\alpha}$ denotes the rotation matrix through angle $\alpha$ :

$$
R_{\alpha}=\left(\begin{array}{rr}
\cos \alpha & -\sin \alpha \\
\sin \alpha & \cos \alpha
\end{array}\right)
$$

$P_{n}, Q_{n}$ are 1-row matrices, $W_{n}$ is a $(2 \times 4)$-matrix. Since $P_{n}, Q_{n}, W_{n}$ are of at least order 1 at $(0,0,0,0)$ and $\left(x_{n}, y_{n}, u_{n}, v_{n}\right)$ decay exponentially fast to $(0,0,0,0)$ as $|n| \rightarrow \infty$, for these matrices the following estimates hold for $|n| \geq N$ and some positive $C$ depending on $N$ and on size of the neighborhood $V$ :

$$
\left\|P_{n}\right\| \leq C \mu_{1}^{|n|}, \quad\left\|Q_{n}\right\| \leq C \mu_{1}^{|n|}, \quad\left\|W_{n}\right\| \leq C \mu_{1}^{|n|},
$$

where $0<\mu<\mu_{1}<1$. Take $|n| \geq N$ and denote

$$
S_{n}=\left(\begin{array}{cc}
E_{2} & 0 \\
0 & R_{n \alpha}
\end{array}\right)
$$

where $E_{2}$ is a $2 \times 2$ identity matrix.

Consider now the case $n \geq N$ and perform in the system (77) a sequence of nonautonomous (with "time" $n$ ) symplectic changes of variables $\zeta_{n}=S_{n-N} \psi_{n}$, where $\psi_{n}=$ $\left(\widehat{\xi}_{n}, \widehat{\eta}_{n}, \widehat{\chi}_{n}\right)$, and consider (7) in the rotating coordinate frame. This change of variables allows one to exclude asymptotically the rotation in coordinates $\chi_{n}=\left(\chi_{n}^{1}, \chi_{n}^{2}\right)$ and prove that in new coordinates each invariant bounded sequence for the linear system obtained from (7) has a limit as $n \rightarrow \infty$.

After the change system (17) casts as follows (we hold previous notation for variables):

$$
\left\{\begin{aligned}
\xi_{n+1} & =\mu \xi_{n}+F_{n} \zeta_{n}, \\
\eta_{n+1} & =\mu^{-1} \eta_{n}+G_{n} \zeta_{n}, \\
\chi_{n+1} & =\chi_{n}+H_{n} \zeta_{n},
\end{aligned}\right.
$$

where $F_{n}, G_{n}$ are again 1-row matrices and $H_{n}$ is a $(2 \times 4)$-matrix. For these matrices estimates similar to those for matrices $P_{n}, Q_{n}$ and $W_{n}$ are valid. Sequence $\left\{\left(\xi_{n}, \eta_{n}, \chi_{n}\right)\right\}$ is called the solution of the system or the invariant sequence if equalities (마) are satisfied for all $n \in \mathbb{Z}_{+}$. The following lemma is valid.

Lemma 3.1. There is an integer $N>0$ large enough such that for any given $\xi^{0} \in \mathbb{R}$, $\chi_{+} \in \mathbb{R}^{2}$ a unique solution $\left\{\left(\xi_{n}, \eta_{n}, \chi_{n}\right)\right\}, n \geq N$, exists for the system (8) such that for this solution the boundary conditions are satisfied: $\xi_{N}=\xi^{0}, \chi_{n} \rightarrow \chi_{+},\left|\xi_{n}\right| \rightarrow 0,\left|\eta_{n}\right| \rightarrow 0$ as $n \rightarrow+\infty$.

Proof. Similar to [24], instead of system (8) consider a system of difference equations (9):

$$
\left\{\begin{array}{l}
\xi_{n}=\mu^{n-N} \xi^{0}+\sum_{s=N}^{n-1} \mu^{n-1-s} F_{s} \zeta_{s}, \\
\eta_{n}=-\sum_{s=n}^{\infty} \mu^{s+1-n} G_{s} \zeta_{s}, \\
\chi_{n}=\chi_{+}-\sum_{s=n}^{\infty} H_{s} \zeta_{s} .
\end{array}\right.
$$

Note that any solution of this system obeys the boundary conditions in the statement of the lemma. Let us show first that the solution of the system (9) is also the solution of the 
system (8) and vice versa. Indeed, the following equalities hold:

$$
\begin{gathered}
\xi_{n+1}=\mu^{n+1-N} \xi^{0}+\sum_{s=N}^{n} \mu^{n-s} F_{s} \zeta_{s} \mu\left(\mu^{n-N} \xi^{0}+\sum_{s=N}^{n-1} \mu^{n-1-s} F_{s} \zeta_{s}\right)+F_{n} \zeta_{n}=\mu \xi_{n}+F_{n} \zeta_{n} \\
\eta_{n+1}=-\sum_{s=n+1}^{\infty} \mu^{s-n} G_{s} \zeta_{s}=G_{n} \zeta_{n}-G_{n} \zeta_{n}-\sum_{s=n+1}^{\infty} \mu^{s-n} G_{s} \zeta_{s} \\
=G_{n} \zeta_{n}+\mu^{-1}\left(-\sum_{s=n}^{\infty} \mu^{s+1-n} G_{s} \zeta_{s}\right)=\mu^{-1} \eta_{n}+G_{n} \zeta_{n}, \\
\chi_{n+1}=\chi_{+}-\sum_{s=n+1}^{\infty} H_{s} \zeta_{s}=\chi_{+}-\sum_{s=n}^{\infty} H_{s} \zeta_{s}+H_{n} \zeta_{n}=\chi_{n}+H_{n} \zeta_{n} .
\end{gathered}
$$

So, if the sequence $\left\{\left(\xi_{n}, \eta_{n}, \chi_{n}\right)\right\}$ solves (9), then it satisfies (8). The converse assertion is given as $n \rightarrow \infty$ by the consecutive application of (8) to an initial point.

Thus, one needs to prove the existence of solutions for system (9). To do this, we use the contraction mapping principle. Denote by $B$ the Banach space of sequences $\zeta=$ $\left\{\left(\xi_{n}, \eta_{n}, \chi_{n}\right)\right\}$ uniformly bounded on $[N,+\infty)$ with the norm

$$
\|\zeta\|=\sup _{n \geq N}\left(\left|\xi_{n}\right|,\left|\eta_{n}\right|,\left\|\chi_{n}\right\|\right) .
$$

The right hand sides of (9) define operator $T: T[\zeta]=\bar{\zeta}$ on $B$. At the first step let us verify that $T$ is defined correctly, that is, $T[B] \subset B$; here $\chi_{+}, \xi^{0}$ and $N$ are considered as parameters. Recall that for $F_{n}, G_{n}, H_{n}$ the following estimates are valid: $\left\|F_{n}\right\|,\left\|G_{n}\right\|,\left\|H_{n}\right\| \leq$ $C \nu^{n}, 0<\nu<1$. Here $C$ depends on $N$ but is finite for a fixed $N$. Denote $\kappa=\max \{\mu, \nu\}<1$. Then one proceeds as follows:

$$
\begin{aligned}
& \left|\bar{\xi}_{n}\right| \leq \kappa^{n-N} \xi^{0}+\|\zeta\| \sum_{s=N}^{n-1} \kappa^{n-1-s} C \kappa^{s}=\kappa^{n-N} \xi^{0}+\|\zeta\| C \kappa^{n-1}(n-N), \\
& \left|\bar{\eta}_{n}\right| \leq\|\zeta\| \sum_{s=n}^{\infty} \kappa^{s+1-n} C \kappa^{s}=C\|\zeta\| \kappa^{1-n} \sum_{s=n}^{\infty} \kappa^{2 s}=C\|\zeta\| \kappa^{1-n} \frac{\kappa^{2 n}}{1-\kappa^{2}}=C\|\zeta\| \frac{\kappa^{n+1}}{1-\kappa^{2}}, \\
& \left\|\bar{\chi}_{n}\right\| \leq\left\|\chi_{+}\right\|+C\|\zeta\| \sum_{s=n}^{\infty} \kappa^{s}=\left\|\chi_{+}\right\|+C\|\zeta\| \frac{\kappa^{n}}{1-\kappa} .
\end{aligned}
$$

Thus, the sequence $\bar{\zeta}$ is uniformly bounded on $[N,+\infty)$, so the operator $T$ is defined correctly.

Next we prove $T$ to be a contraction map:

$$
\begin{gathered}
\left|\bar{\xi}_{n}^{1}-\bar{\xi}_{n}^{2}\right| \leq\left\|\zeta_{1}-\zeta_{2}\right\| C \sum_{s=N}^{n-1} \kappa^{n-1-s} \kappa^{s}=\left\|\zeta_{1}-\zeta_{2}\right\| C \kappa^{n-1}(n-N), \\
\left|\bar{\eta}_{n}^{1}-\bar{\eta}_{n}^{2}\right| \leq\left\|\zeta_{1}-\zeta_{2}\right\| C \sum_{s=n}^{\infty} \kappa^{s+1-n} \kappa^{s}=\left\|\zeta_{1}-\zeta_{2}\right\| C \kappa^{1-n} \frac{\kappa^{2 n}}{1-\kappa^{2}} \\
=\left\|\zeta_{1}-\zeta_{2}\right\| \frac{C \kappa^{n+1}}{1-\kappa^{2}} \leq\left\|\zeta_{1}-\zeta_{2}\right\| \frac{C \kappa^{N}}{1-\kappa^{2}}, \\
\left\|\bar{\chi}_{n}^{1}-\bar{\chi}_{n}^{2}\right\| \leq\left\|\zeta_{1}-\zeta_{2}\right\| C \sum_{s=n}^{\infty} \kappa^{s}=\left\|\zeta_{1}-\zeta_{2}\right\| \frac{C \kappa^{n}}{1-\kappa} \leq\left\|\zeta_{1}-\zeta_{2}\right\| \frac{C \kappa^{N}}{1-\kappa} .
\end{gathered}
$$


These estimates show that $T$ is contracting for $N$ large enough and $n \geq N$. Thus, for any fixed $\xi^{0} \in \mathbb{R}$ and $\chi_{+} \in \mathbb{R}^{2}$ there is a unique solution

$$
\zeta\left(\xi^{0}, \chi_{+}\right)=\left\{\zeta_{n}\left(\xi^{0}, \chi_{+}\right)\right\}=\left\{\left(\xi_{n}\left(\xi^{0}, \chi_{+}\right), \eta_{n}\left(\xi^{0}, \chi_{+}\right), \chi_{n}\left(\xi^{0}, \chi_{+}\right)\right)\right\}
$$

for the system (9) such that $\xi_{N}\left(\xi^{0}, \chi_{+}\right)=\xi^{0}$. The estimates above also show that $\left|\xi_{n}\right|$, $\left|\eta_{n}\right|$ and $\left\|\chi_{n}-\chi_{+}\right\|$tend to zero as $n \rightarrow \infty$.

For further purposes one needs to prove some linearity relations for solutions of system (8).

Lemma 3.2. Solutions $\zeta\left(\xi^{0}, \chi_{+}\right)$of the system (8) satisfy the following linearity relations:

I. $\zeta\left(\xi_{0}^{\prime}, \chi_{+}\right)+\zeta\left(\xi_{0}^{\prime \prime}, 0\right)=\zeta\left(\xi_{0}^{\prime}+\xi_{0}^{\prime \prime}, \chi_{+}\right)$;

II. $\zeta\left(\xi_{0}^{\prime}, 0\right)+\zeta\left(\xi_{0}^{\prime \prime}, 0\right)=\zeta\left(\xi_{0}^{\prime}+\xi_{0}^{\prime \prime}, 0\right), \zeta\left(\alpha \xi_{0}^{\prime}, 0\right)=\alpha \zeta\left(\xi_{0}^{\prime}, 0\right)$;

III. $\zeta\left(0, \alpha \chi_{+}+\beta \chi_{+}^{\prime \prime}\right)=\alpha \zeta\left(0, \chi_{+}^{\prime}\right)+\beta \zeta\left(0, \chi_{+}^{\prime \prime}\right)$.

Proof. To prove the first equality consider the function

$$
\begin{aligned}
\Delta_{1}=\left\{\Delta_{1}(n)\right\}=\zeta\left(\xi_{0}^{\prime}, \chi_{+}\right)+\zeta & \left(\xi_{0}^{\prime \prime}, 0\right)-\zeta\left(\xi_{0}^{\prime}+\xi_{0}^{\prime \prime}, \chi_{+}\right) \\
= & \left\{\left(\begin{array}{c}
\xi_{n}\left(\xi_{0}^{\prime}, \chi_{+}\right)+\xi_{n}\left(\xi_{0}^{\prime \prime}, 0\right)-\xi_{n}\left(\xi_{0}^{\prime}+\xi_{0}^{\prime \prime}, \chi_{+}\right) \\
\eta_{n}\left(\xi_{0}^{\prime}, \chi_{+}\right)+\eta_{n}\left(\xi_{0}^{\prime \prime}, 0\right)-\eta_{n}\left(\xi_{0}^{\prime}+\xi_{0}^{\prime \prime}, \chi_{+}\right) \\
\chi_{n}\left(\xi_{0}^{\prime}, \chi_{+}\right)+\chi_{n}\left(\xi_{0}^{\prime \prime}, 0\right)-\chi_{n}\left(\xi_{0}^{\prime}+\xi_{0}^{\prime \prime}, \chi_{+}\right)
\end{array}\right)\right\} .
\end{aligned}
$$

This function is a solution of the system (9) with boundary conditions $(0,0)$. Indeed, consider the following systems with boundary conditions $\left(\xi_{0}^{\prime}, \chi_{+}\right),\left(\xi_{0}^{\prime \prime}, 0\right)$ and $\left(\xi_{0}^{\prime}+\xi_{0}^{\prime \prime}, \chi_{+}\right)$, respectively:

$$
\begin{aligned}
& \left\{\begin{array}{l}
\xi_{n}\left(\xi_{0}^{\prime}, \chi_{+}\right)=\mu^{n-N} \xi^{0}+\sum_{s=N}^{n-1} \mu^{n-1-s} F_{s} \zeta_{s}\left(\xi_{0}^{\prime}, \chi_{+}\right) \\
\eta_{n}\left(\xi_{0}^{\prime}, \chi_{+}\right)=-\sum_{s=n}^{+\infty} \mu^{s+1-n} G_{s} \zeta_{s}\left(\xi_{0}^{\prime}, \chi_{+}\right) \\
\chi_{n}\left(\xi_{0}^{\prime}, \chi_{+}\right)=\chi_{+}-\sum_{s=n}^{+\infty} H_{s} \zeta_{s}\left(\xi_{0}^{\prime}, \chi_{+}\right)
\end{array}\right. \\
& \left\{\begin{array}{l}
\xi_{n}\left(\xi_{0}^{\prime \prime}, 0\right)=\mu^{n-N} \xi^{0}+\sum_{s=N}^{n-1} \mu^{n-1-s} F_{s} \zeta_{s}\left(\xi_{0}^{\prime \prime}, 0\right) \\
\eta_{n}\left(\xi_{0}^{\prime \prime}, 0\right)=-\sum_{s=n}^{+\infty} \mu^{s+1-n} G_{s} \zeta_{s}\left(\xi_{0}^{\prime \prime}, 0\right) \\
\chi_{n}\left(\xi_{0}^{\prime \prime}, 0\right)=\chi_{+}-\sum_{s=n}^{+\infty} H_{s} \zeta_{s}\left(\xi_{0}^{\prime \prime}, 0\right)
\end{array}\right. \\
& \left\{\begin{array}{l}
\xi_{n}\left(\xi_{0}^{\prime}+\xi_{0}^{\prime \prime}, \chi_{+}\right)=\mu^{n-N} \xi^{0}+\sum_{s=N}^{n-1} \mu^{n-1-s} F_{s} \zeta_{s}\left(\xi_{0}^{\prime}+\xi_{0}^{\prime \prime}, \chi_{+}\right), \\
\eta_{n}\left(\xi_{0}^{\prime}+\xi_{0}^{\prime \prime}, \chi_{+}\right)=-\sum_{s=n}^{+\infty} \mu^{s+1-n} G_{s} \zeta_{s}\left(\xi_{0}^{\prime}+\xi_{0}^{\prime \prime}, \chi_{+}\right), \\
\chi_{n}\left(\xi_{0}^{\prime}+\xi_{0}^{\prime \prime}, \chi_{+}\right)=\chi_{+}-\sum_{s=n}^{+\infty} H_{s} \zeta_{s}\left(\xi_{0}^{\prime}+\xi_{0}^{\prime \prime}, \chi_{+}\right) .
\end{array}\right.
\end{aligned}
$$


Summing related equalities from (10), (11) and subtracting (12) we get:

$$
\begin{gathered}
\xi_{n}\left(\xi_{0}^{\prime}, \chi_{+}\right)+\xi_{n}\left(\xi_{0}^{\prime \prime}, 0\right)-\xi_{n}\left(\xi_{0}^{\prime}+\xi_{0}^{\prime \prime}, \chi_{+}\right)=\mu^{n-N}\left(\xi_{0}^{\prime}+\xi_{0}^{\prime \prime}-\left(\xi_{0}^{\prime}+\xi_{0}^{\prime \prime}\right)\right) \\
+\sum_{s=N}^{n-1} \mu^{n-s-1} F_{s}\left(\zeta_{s}\left(\xi_{0}^{\prime}, \chi_{+}\right)+\zeta_{s}\left(\xi_{0}^{\prime \prime}, 0\right)-\zeta_{s}\left(\xi_{0}^{\prime}+\xi_{0}^{\prime \prime}, \chi_{+}\right)\right)=\sum_{s=N}^{n-1} \mu^{n-s-1} F_{s} \Delta_{1}(s), \\
\eta_{n}\left(\xi_{0}^{\prime}, \chi_{+}\right)+\eta_{n}\left(\xi_{0}^{\prime \prime}, 0\right)-\eta_{n}\left(\xi_{0}^{\prime}+\xi_{0}^{\prime \prime}, \chi_{+}\right) \\
=-\sum_{s=n}^{+\infty} \mu^{-(n-s-1)} G_{s}\left(\zeta_{s}\left(\xi_{0}^{\prime}, \chi_{+}\right)+\zeta_{s}\left(\xi_{0}^{\prime \prime}, 0\right)-\zeta_{s}\left(\xi_{0}^{\prime}+\xi_{0}^{\prime \prime}, \chi_{+}\right)\right) \\
\chi_{n}\left(\xi_{0}^{\prime}, \chi_{+}\right)+\chi_{n}\left(\xi_{0}^{\prime \prime}, 0\right)-\chi_{n}\left(\xi_{0}^{\prime}+\xi_{0}^{\prime \prime}, \chi_{+}\right)=\left(\chi_{+}+0-\chi_{+}\right) \\
-\sum_{s=n}^{+\infty} H_{s}\left(\zeta_{s}\left(\xi_{0}^{\prime}, \chi_{+}\right)+\zeta_{s}\left(\xi_{0}^{\prime \prime}, 0\right)-\zeta_{s}\left(\xi_{0}^{\prime}+\xi_{0}^{\prime \prime}, \chi_{+}\right)\right)-\sum_{s=n}^{+\infty} H_{s} \Delta_{1}(s) .
\end{gathered}
$$

The equalities obtained imply that $\Delta_{1}$ satisfies (9) with boundary conditions $\left(\xi^{0}, \chi_{+}\right)=(0,0)$. Since the solution of (9) with given boundary conditions is unique, then $\Delta_{1} \equiv 0$ and therefore the relation I is valid.

Relations II and III are proved in a similar way if instead of $\Delta_{1}$ one considers $\Delta_{2}$ and $\Delta_{3}$, respectively:

$$
\begin{aligned}
& \Delta_{2}=\zeta\left(\alpha \xi_{0}^{\prime}+\beta \xi_{0}^{\prime \prime}, 0\right)-\alpha \zeta\left(\xi_{0}^{\prime}, 0\right)-\beta \zeta\left(\xi_{0}^{\prime \prime}, 0\right), \\
& \Delta_{3}=\zeta\left(0, \alpha \chi_{+}^{\prime}+\beta \chi_{+}^{\prime \prime}\right)-\alpha \zeta\left(0, \chi_{+}^{\prime}\right)-\beta \zeta\left(0, \chi_{+}^{\prime \prime}\right) .
\end{aligned}
$$

The lemma has been proved.

Similar constructions are done for $n \leq-N$.

3.1. Geometry of linearized map. Now let us present a geometrical interpretation of the results obtained. To do this, we introduce a countable set of linear symplectic spaces $X_{n},|n| \geq N$, with coordinates $\left(\xi_{n}, \eta_{n}, \chi_{n}^{1}, \chi_{n}^{2}\right)$, and linear symplectic maps $\mathcal{L}_{n}: X_{n} \rightarrow X_{n+1}, n \geq N$, defined by (9). If we fix $\chi_{+}$and vary $\xi_{0}, \xi_{0}=\xi_{0}^{\prime}+\xi_{0}^{\prime \prime}, \xi_{0}^{\prime \prime} \in \mathbb{R}$, then due to Lemma 3.2, the related solutions of (9) define an affine straight line in $X_{N}$ (in fact, they are initial points of these solutions) and hence in any $X_{n}, n>N$. These straight lines have the characteristic property that any solution which passes through this line in $X_{n}$ decays exponentially as $n \rightarrow \infty: \xi_{n} \rightarrow 0, \eta_{n} \rightarrow 0,\left\|\chi_{n}-\chi_{+}\right\| \rightarrow 0$. In addition, if we fix not $\chi_{+}$but only the value $2 I=\chi_{1}^{2}+\chi_{2}^{2}=\left\|\chi_{+}\right\|^{2}$, then in every $X_{n}$, $n \geq N$, we get a 2-dimensional cylinder $C_{n}^{+}(I)$ formed of those straight lines in $X_{n}$ through which solutions asymptotically satisfy $\left\|\chi_{n}-\chi_{+}\right\| \rightarrow 0$, and each straight line on $C_{n}^{+}(I)$ corresponds to a unique point on the circle $2 I=\chi_{1}^{2}+\chi_{2}^{2}$ (an asymptotic phase). Varying $I, \xi_{0}$ defines a linear 3-dimensional subspace $\mathcal{L}_{n}^{+}$of bounded solutions in $X_{N}$, and hence in $X_{n}$, which in turn foliates on the cylinders $C_{n}^{+}(I)$. Such a cylinder shrinks to the straight line $C_{n}^{+}(0)$, as $I \rightarrow 0$; this straight line just corresponds to solutions with $\chi_{+}=0$.

Now let us turn to the initial linearization problem along homoclinic orbit $\Gamma$ for diffeomorphism $f$. To derive results described above, we performed the sequence of linear changes of variables that allowed us to prove for any bounded solution the existence of an asymptotic phase. In the initial coordinates all objects found preserve: for any point $q_{n}$ in the related tangent space $T_{q_{n}} M$ we have a 3 -dimensional subspace of bounded as $n \rightarrow \infty$ solutions $\mathcal{L}_{n}^{+}$(we preserve the same notation for similar objects) which are foliated into cylinders $C_{n}^{+}(I)$ (it is worth mentioning that the value of $I$ does not change when 


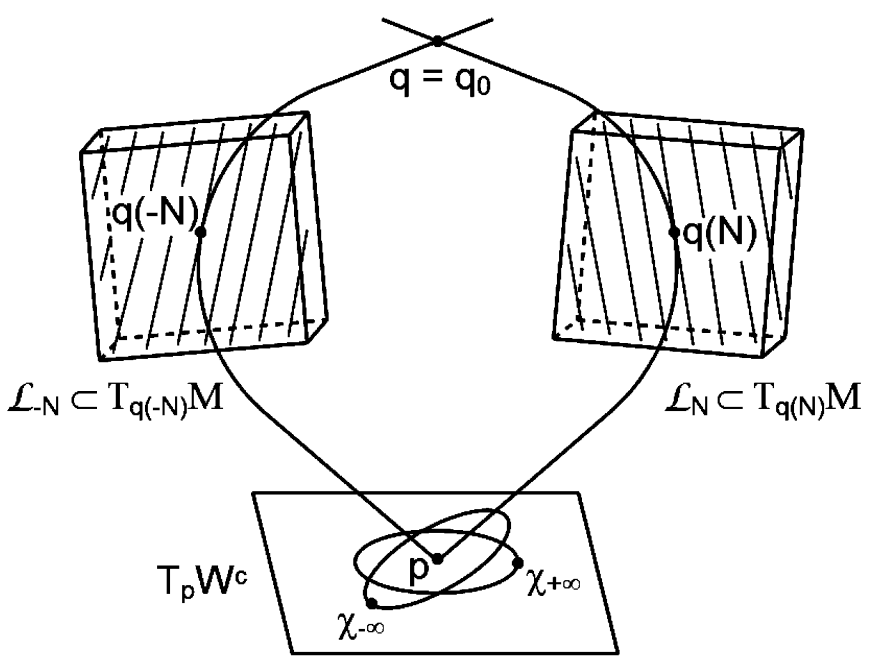

FiguRE 2. Scattering map construction

returning to the initial coordinates), foliations into straight lines, etc. It is evident that in fact $\mathcal{L}_{n}^{+}$is nothing more than tangent space $T_{q_{n}} W^{c s}$.

The same picture takes place for $X_{n}$ with $n \leq-N$; the only difference is that one needs to take limits as $n \rightarrow-\infty$. Here we also have cylinders $C_{n}^{-}$, straight lines, 3 -subspaces $\mathcal{L}_{n}^{-}=T_{q_{n}} W^{c u}$, and so forth.

For tangent space $T_{q(-N)} M$ and $T_{q(N)} M$ we have the linear symplectic map

$$
\mathcal{S}=D f^{2 N}: T_{q(-N)} M \rightarrow T_{q(N)} M
$$

calculated at the point $q(-N)$. This map transforms $\mathcal{L}_{n}^{-}$to a 3 -dimensional subspace in $T_{q(N)} M$ which transversely intersects the straight line $C_{N}^{+}(0)=l_{N}^{s}$ being the tangent space to $W^{s}(p)$.

3.2. Scattering map. Now we are ready to construct the scattering map

$$
S: T_{p} W^{c} \rightarrow T_{p} W^{c} .
$$

Take any point $s \in T_{p} W^{c}$. Fixing this point defines the unique straight line in $T_{q(-N)} M$ of the foliation defined in $\mathcal{L}_{-N}=T_{q(-N)} W^{c u}$ whose points are asymptotic to $s$ as $n \rightarrow-\infty$. Let us apply linear map $D f^{2 N}$ to points of this line. We get the straight line in $T_{q(N)} M$ which is transversal to 3 -plane $\mathcal{L}_{N}=T_{q(N)} W^{c s}$ due to the transversality condition. Thus, the line obtained intersects this 3 -plane at the unique point through which a unique line of the foliation defined in plane $\mathcal{L}_{N}$ passes. Denote by $s_{1} \in T_{p} W^{c}$ that unique point which is the limit as $n \rightarrow \infty$ for all sequences starting on this line. We set $S(s)=s_{1}$ (Figure 2).

Let us verify that $S$ is a linear map. It is clear that $S(0)=0$. Indeed, for $s=0$ the corresponding straight line in 3-plane $\mathcal{L}_{-N}$ is the tangent line to $W^{u}$ in tangent space $T_{q(-N)} M$. Its image under $\mathcal{S}=D f^{2 N}$ is a straight line in $T_{q(N)} M$ which is transversal to $T_{q(N)} W^{c s}$ due to Assumption 2 and intersects it at the origin of $T_{q(N)} M$. Through the origin the unique line from the constructed foliation passes: the tangent line to $W^{s}$ which corresponds to $s_{1}=0$ in $T_{p} W^{c}$.

Denote by $l_{s}$ that straight line in $\mathcal{L}_{-N}$ which consists of points through which solutions pass tending to $s$ as $n \rightarrow-\infty$. Using the linearity relations I and II we get the following representation for the solutions of system (8):

$$
\zeta_{n}\left(\xi^{0}, \lambda \chi_{-}\right)=\zeta_{n}\left(0, \lambda \chi_{-}\right)+\zeta_{n}\left(\xi^{0}, 0\right)=\lambda \zeta_{n}\left(0, \chi_{-}\right)+\zeta_{n}\left(\xi^{0}, 0\right) \quad \text { for any } \lambda \in \mathbb{R} .
$$


To find the image $S(\lambda s)$ we act by $D f^{2 N}$ on $\lambda l_{s}+l_{0}^{u}$ (here $l_{0}^{u}$ is the tangent line to $W^{u}$ at point $q(-N))$. In $\mathcal{L}_{N}$ we get two vectors; through each such vector a unique straight line passes: these lines are $\lambda l_{s}$ and $l_{0}^{s}$, respectively $\left(l_{0}^{s}\right.$ is a tangent line to $W^{s}$ at point $\left.q_{N}\right)$. Thus, $S$ maps $\lambda s$ to $\lambda s_{1}+0=\lambda s_{1}$. Similarly, for the sum $s^{\prime}+s^{\prime \prime}$ we get relations for corresponding $\chi_{-}^{\prime}, \chi_{-}^{\prime \prime}$ :

$$
\begin{aligned}
\zeta_{n}\left(\xi^{0}, \chi_{-}^{\prime}+\chi_{-}^{\prime \prime}\right) & =\zeta_{n}\left(\xi^{0}+0, \chi_{-}^{\prime}+\chi_{-}^{\prime \prime}\right) \\
& =\zeta_{n}\left(\xi^{0}, 0\right)+\zeta_{n}\left(0, \chi_{-}^{\prime}+\chi_{-}^{\prime \prime}\right)=\zeta_{n}\left(\xi^{0}, 0\right)+\zeta_{n}\left(0, \chi_{-}^{\prime}\right)+\zeta_{n}\left(0, \chi_{-}^{\prime \prime}\right) .
\end{aligned}
$$

In this case we act by $D f^{2 N}$ on $l_{s^{\prime}}+l_{s^{\prime \prime}}+l_{0}^{u}$.

The next proposition characterizes map $S$.

Proposition 3.3. Map $S: T_{p} W^{c} \rightarrow T_{p} W^{c}$ is symplectic.

Proof. Choose any two vectors in the symplectic plane $T_{p} W^{c}$. These vectors define two straight lines from the foliation in $\mathcal{L}_{-N}$. Take two vectors $v_{1}, v_{2}$ in $\mathcal{L}_{-N}=T_{q(-N)} W^{c u}$ corresponding to these lines: origins of vectors coincide with zero point of $T_{q(-N)} W^{c u}$, and ends of the vectors belong to the corresponding line. The skew-scalar product being the restriction of 2 -form $\Omega$ on tangent space $\mathcal{L}_{-N}$ does not depend on the vectors we choose. Indeed, the difference of vectors corresponding to the same line is the vector lying in $l_{-}^{u}$ which is the zero vector for the skew-scalar product (such vectors shrink exponentially in backward iterations). $\mathcal{S}$-images of these two straight lines are two straight lines in $T_{q(N)} M$ which are transversal to subspace $\mathcal{L}_{N}$. The intersection of the lines with $\mathcal{L}_{N}$ gives two vectors $T\left(v_{1}\right), T\left(v_{2}\right)$, whose origins coincide with zero of $\mathcal{L}_{N}=T_{q(N)} W^{c s}$ for specified $v_{1}, v_{2}$. Since $\mathcal{S}$ is a linear symplectic map, then the skew-scalar product is preserved. Now we have two straight lines from foliation in $\mathcal{L}_{N}$, and again the skewscalar product of vectors corresponding to different lines does not depend on the exact vectors we choose. But this product is equal to the skew-scalar product of vectors $v_{1}, v_{2}$ and does not change in forward iterations. Therefore the skew-scalar product in limit in $T_{p} W^{c}$ equals the skew-scalar product of initial vectors in $T_{p} W^{c}$.

The linear symplectic map $S$ we call the scattering map.

\section{Homoclinic orbits to invariant KAM-CURVES}

To prove Theorem 1.1 we assume for diffeomorphism $f$ Assumptions 1, 2 and 3 to hold. Thus, according to Section 2 manifolds $W^{c u}(p)$ and $W^{c s}(p)$ intersect at homoclinic point $q$ transversally and therefore along a symplectic 2-disk $\Sigma$ (we may regard $q=q_{+}$ and disk $\Sigma$ as belonging to a neighborhood of fixed point $p$ ). The idea of the existence proof for homoclinic orbits to invariant KAM-curve $\gamma \in W^{c}$ is the following. Let $V$ be a sufficiently small neighborhood of point $p$. For each KAM-curve $\gamma \subset W^{c} \cap V$ its action is defined according to the Stokes theorem as the integral of 2 -form $\Omega$ over that disk in $W^{c}$ whose boundary is curve $\gamma$. Curve $\gamma$ has its local stable manifold which can be extended by a finite number of iterations of map $f^{-1}$ till the manifold reaches the neighborhood of homoclinic point $q$ staying inside of $W^{c s}(p)$. Therefore, this manifold intersects transversally within $W^{c s}(p)$ disk $\Sigma$ along a closed curve $w_{s}(\gamma)$. Similarly, the unstable manifold of the same curve $\gamma$ under the action of $f$ reaches the neighborhood of $q$ staying inside $W^{c u}(p)$ and hence intersects $\Sigma$ along a closed curve $w_{u}(\gamma)$. Two obtained curves on $\Sigma$ have the same value of action as follows from Lemma 2.2 Thus, two disks in $\Sigma$ bounded by $w_{s}(\gamma), w_{u}(\gamma)$ are of the same area and have common point $q$ lying inside both of them. Hence, the intersection of curves $w_{s}(\gamma)$ and $w_{u}(\gamma)$ is not empty and consists of at least two different points, and homoclinic orbits to $\gamma$ pass through the intersection points (see Figure 3). 


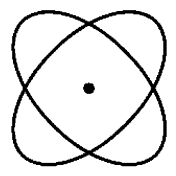

(1)

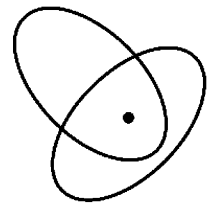

(2)

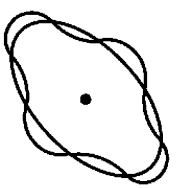

(3)

Figure 3. Possible intersections of $W^{s}(\gamma), W^{u}(\gamma)$ on $\Sigma$

The problem here is that we do not know precise information on this intersection: how many points it contains, if the intersection is transverse or not, etc. All these questions are relevant for the further study of nearby dynamics. In the case of an integrable diffeomorphism (when an invariant w.r.t. the diffeomorphism $f$ smooth function exists) these two curves coincide, since both of them belong to the same level of the invariant function whose restriction on $\Sigma$ near $q$ usually forms a connected closed curve.

Provided that our assumptions hold, we shall prove for any KAM-curve on $W^{c}$ in a sufficiently small $V$ with a given value of action $I$ that the intersection consists of exactly four points and it is transverse at each of these points. To prove this we shall connect intersection properties for curves $w_{u}(\gamma)$ and $w_{s}(\gamma)$ on $\Sigma$ with intersection properties of related ellipses $E$ and $S(E)$ with the same action $I$ in tangent plane $T_{p} W^{c}$. The genericity assumption implies that these ellipses have the same center and the same area and intersect transversally at exactly four points. This property will be carried to the intersection of $w_{u}(\gamma)$ and $w_{s}(\gamma)$.

To prove the intersection of these curves is as in Figure 3 (left panel), we transform $f$ in neighborhood $V$ of fixed point $p$ to the normal form (15) (see Appendix 7.2) up to the third order terms and consider first the truncated map. For this map two local functions $x y$ and $u^{2}+v^{2}$ are local integrals up to sixth order terms, but what is more important for our goals, circles $x=y=0, u^{2}+v^{2}=c$ on $W^{c}$ are invariant curves for any positive $c$ small enough and stable and unstable manifolds of these curves have the representation $y=0, u^{2}+v^{2}=c$ and $x=0, u^{2}+v^{2}=c$, respectively. Since the whole map differs from the truncated map by terms of the fourth order and higher, then for a given positive constant $c$ small enough invariant manifolds of KAM-curves for the truncated and full maps are at least $C^{3}$-close in $V$, due to Fenichel theorems. According to these theorems [15], as a diffeomorphism is $C^{r}$-smooth, then invariant cylinders of KAM-curves are $C^{r-1}$-smooth (see Appendix (7.3). Also we shall suppose, without loss of generality, that both points $q_{-}=q(-N)$ and $q_{+}=q(N)$ belong to $V$.

For diffeomorphism (15) for $n \geq N$, due to properties of functions $\varphi, \psi$, system (8) has the linearization matrices for $f$ along the homoclinic orbit

$$
\left(\begin{array}{cccc}
\mu(1+\ldots) & p_{n} & q_{n} & r_{n} \\
0 & \mu^{-1}(1+\ldots) & 0 & 0 \\
0 & l_{n} & \cos \alpha+\ldots & -\sin \alpha+\ldots \\
0 & s_{n} & \sin \alpha+\ldots & \cos \alpha+\ldots
\end{array}\right)
$$

where dots and $p_{n}, q_{n}, r_{n}, l_{n}, s_{n}$ mean terms tending to zero exponentially fast as $n \rightarrow+\infty$. The form of this matrix implies that 3 -dimensional plane $\eta=0$ in the tangent space at homoclinic point $q(N)$ under the action of this linear map is transformed to 3-dimensional plane $\eta=0$ in the tangent space at homoclinic points $q(N+1)=f(q(N))$, etc. As Lemma 3.1 implies, for a fixed $I,\left(\chi^{1}\right)^{2}+\left(\chi^{2}\right)^{2}=2 I$, we get in $T_{q(N)} M$ a cylinder in 3-dimensional plane $\eta=0$, consisting of solutions for the system (8) which asymptotically tend to the circle $\left(\chi^{1}\right)^{2}+\left(\chi^{2}\right)^{2}=2 I$ in $T_{p} W^{c}$. Intersection of this cylinder with tangent 
plane to $\Sigma_{+}$at point $q(N)$ is an ellipse. Similar cylinders and ellipses are obtained if one considers linearization of the diffeomorphism along homoclinic orbit for $n \leq-N$ as $n \rightarrow-\infty$.

Global symplectic map $\mathcal{G}=f^{2 N}$ transforms a neighborhood of homoclinic point $q(-N)$ into a neighborhood of homoclinic point $q(N)$. In normalized coordinates (15), homoclinic points have the coordinates

$$
q(-N)=\left(0, y_{1}, 0,0\right), \quad q(N)=\left(x_{0}, 0,0,0\right) .
$$

Therefore, symplectic map $\mathcal{G}$ has the following local representation:

$$
\begin{gathered}
\bar{x}-x_{0}=F\left(x, y-y_{1}, u, v\right), \quad \bar{y}=G\left(x, y-y_{1}, u, v\right), \\
\bar{u}=P\left(x, y-y_{1}, u, v\right), \quad \bar{v}=Q\left(x, y-y_{1}, u, v\right), \\
d \bar{x} \wedge d \bar{y}+d \bar{u} \wedge d \bar{v}=d x \wedge d y+d u \wedge d v,
\end{gathered}
$$

and all functions $F, G, P, Q$ vanish at the point $\left(0, y_{1}, 0,0\right)$. The transversality condition at point $q(N)$ means the tangent vector to $W^{s}$, that is, $(1,0,0,0)$, is transverse to the tangent plane to $W^{c u}$, which is the $\mathcal{G}$-image of the 3 -disk $x=0$. This implies that determinant

$$
\operatorname{det}\left(\frac{\partial(G, P, Q)}{\partial\left(y-y_{1}, u, v\right)}\right)
$$

calculated at point $\left(0, y_{1}, 0,0\right)$ does not vanish. By virtue of Lemma 2.1 the same transversality condition holds at $q(-N)$ : the tangent vector to $W^{u}$ (i.e. $(0,1,0,0)$ ) and the tangent plane to $W^{c s}$ (the $\mathcal{G}$-pre-image of $\bar{y}=0$ ) are transverse; this is equivalent to the inequality $G_{y} \neq 0$ at $\left(0, y_{1}, 0,0\right)$. The 2-Disk $\Sigma=\Sigma_{+}$at these coordinates is the intersection of the $\mathcal{G}$-image of local 3-disk $x=0$ near point $q(-N)$ and local 3-disk $\bar{y}=0$ near $q(N)$. Then one has a representation for $\Sigma_{-}=\mathcal{G}^{-1}\left(\Sigma_{+}\right)$in the form $x=0, y-y_{1}=\Psi(u, v)$, and for $\Sigma_{+}$in the form $\bar{x}=\Phi(\bar{u}, \bar{v}), \bar{y}=0$. In particular, since map $\mathcal{G}$ is symplectic and the restrictions of symplectic 2-form $\Omega$ to symplectic disks $\Sigma_{-}$and $\Sigma_{+}$are $d u \wedge d v$ and $d \bar{u} \wedge d \bar{v}$, respectively, then the restriction $\mathcal{G}_{\Sigma}$ of map $\mathcal{G}, \mathcal{G}_{\Sigma}: \Sigma_{-} \rightarrow \Sigma_{+}$is symplectic and has the form

$$
\bar{u}=a_{11} u+a_{12} v+\ldots, \quad \bar{v}=a_{21} u+a_{22} v+\ldots,
$$

where dots mean terms of the second order and higher, and the matrix

$$
A=\left(\begin{array}{ll}
a_{11} & a_{12} \\
a_{21} & a_{22}
\end{array}\right)
$$

is symplectic; here this means uni-modular: $\operatorname{det} A=1$. In fact, matrix $A$ depends on integer parameter $N, A=A_{N}$, since our choice of the homoclinic points $q(-N), q(N)$ and hence $\Sigma_{-}, \Sigma_{+}$, and related tangent planes to them depends on $N$. But for any $N$ large enough the representations for these tangent planes are similar, and coordinates of them are coordinates on $T_{p} W^{c}$. As $N \rightarrow \infty$ the related tangent planes to $\Sigma_{+}(N)$ tend to $T_{p} W^{c}$. This implies

Lemma 4.1. For $N$ large enough matrix $A_{N}$ is not a rotation matrix.

Proof. To prove Lemma 4.1 we shall show that its conclusion follows from the transversality Assumption 2 and genericity Assumption 3 . As follows from Lemmas $3.1,3.2, A_{N}$ tends to matrix $A_{\infty}$ being the coordinate representation of scattering map $S$.

Consider symplectic disk $\Sigma_{-}$through the point $q(-N)$. In normalized coordinates it has a representation $x=0, y=\Psi(u, v)$. Hence the tangent plane to it at $q(-N)$ has a representation $\xi=0, \eta=a \chi^{1}+b \chi^{2}$. This implies this plane intersects transversely any Lagrangian cylinder $C_{n}^{-}(I)$, and these intersections form a foliation of the plane into ellipses. Similar foliation into ellipses exists in the tangent plane to $\Sigma_{+}$through 
the point $q(N)$; it is generated by intersection of this plane with cylinders $C_{n}^{+}(I)$. The differential of the global map $\mathcal{G}=f^{2 N}$ is a linear symplectic map, and its restriction to $\Sigma_{-}$ is a linear symplectic map $L_{N}: T_{q(-N)} \Sigma_{-} \rightarrow T_{q(N)} \Sigma_{+}$. If we fix $I$, then related ellipses $E_{-}(I), E_{+}(I)$ in $\Sigma_{-}, \Sigma_{+}$, respectively, have the same area and $L_{N}\left(E_{-}(I)\right)$ intersects $E_{+}(I)$ at four different points transversely, due to Assumption 3 .

Let us fix a sufficiently small neighborhood of fixed point $p$ on the center manifold $W^{c}$. Its smallness is controlled by a parameter $\varepsilon$. To this end, following [36], we introduce symplectic polar coordinates on $W^{c}=\{x=y=0\}$ in the neighborhood of fixed points

$$
u=\varepsilon \sqrt{2 I} \cos \theta, \quad v=\varepsilon \sqrt{2 I} \sin \theta .
$$

Then we get a symplectic map on $W^{c}$ :

$$
\bar{I}=I+\mathcal{O}\left(\varepsilon^{3}\right), \quad \bar{\theta}=\theta+\alpha+\nu \varepsilon^{2} I+\mathcal{O}\left(\varepsilon^{3}\right) .
$$

According to the Moser theorem [36], there is an $I_{0}>0$ such that for $\varepsilon$ small enough and any given $I, 0<I<I_{0}$, such that the number $\alpha+2 \nu \varepsilon^{2} I$ is Diophantine, there exists an invariant curve which is $\varepsilon^{2}$-close in $C^{2}$ topology in the space of curves $I=r(\theta)$ to curve $u^{2}+v^{2}=2 \varepsilon^{2} I$. The map (14) is the restriction of the initial map on a neighborhood of $p$ in $W^{c}$ and normalized up to the third order terms. Thus each invariant curve on the center manifold has two invariant Lagrangian cylinders being its stable and unstable manifolds; they are $C^{2}$-close to cylinders $u^{2}+v^{2}=2 \varepsilon^{2} I, y=0$, or $x=0$, respectively, of the truncated map.

Let us verify that traces of $W^{s}(\gamma), W^{u}(\gamma)$ corresponding to invariant KAM-curve $\gamma$ on $T_{p} W^{c}$ with action $2 \varepsilon^{2} I$ also intersect on $\Sigma_{+}$transversally along four points. To this purpose, we consider the restriction of $\mathcal{G}$ on $\Sigma_{-}$near point $q(-N)$ with values on disk $\Sigma_{+}$ near point $q(N)$. Fix in $V$ some neighborhood of $p$ on $W^{c}$ defined by $\varepsilon$ small enough, and let $\gamma$ be some KAM-curve in this neighborhood. This defines some $I$. The restriction of $\mathcal{G}$ on $\Sigma_{-}$is a 2-dimensional symplectic map (13). Since the coordinates on $\Sigma_{-}$and $\Sigma_{+}$ are $(u, v)$, after the change of variables (14) where $\varepsilon$ and $I$ are considered as parameters, we come to the system for intersection points of $w^{s}(\gamma), w^{u}(\gamma)$. Taking into account that these manifolds are of the same KAM-curve, we get that the value $I$ is the same and then we have

$$
\begin{aligned}
& \varepsilon \sqrt{2 I} \cos \bar{\theta}=\varepsilon a_{11} \sqrt{2 I} \cos \theta+\varepsilon a_{12} \sqrt{2 I} \sin \theta+\mathcal{O}\left(\varepsilon^{2}\right), \\
& \varepsilon \sqrt{2 I} \sin \bar{\theta}=\varepsilon a_{21} \sqrt{2 I} \cos \theta+\varepsilon a_{22} \sqrt{2 I} \sin \theta+\mathcal{O}\left(\varepsilon^{2}\right) .
\end{aligned}
$$

Dividing the equations on $\varepsilon \sqrt{2 I}$, squaring both sides of each of the equalities and summing them we get the equation for $\theta$ corresponding intersection points:

$$
1=\left(a_{11} \cos \theta+a_{12} \sin \theta\right)^{2}+\left(a_{21} \cos \theta+a_{22} \sin \theta\right)^{2}+\mathcal{O}(\varepsilon) .
$$

This equation has precisely four simple roots if matrix $A$ is not a rotation matrix [30. Therefore, due to the implicit function theorem, traces of cylinders of the truncated map on disk $\Sigma$ intersect transversally along four points. This implies that $C^{2}$-close traces of cylinders of the full map, that is, curves $w_{u}(\gamma)$ and $w_{s}(\gamma)$, also intersect transversally along four points. Theorem 1.1 has been proved.

Results given in Theorem 1.1 have much wider applications than are formulated there. Indeed, let us fix some invariant KAM-curve $\gamma$ on $W^{c}$ being sufficiently close to fixed point $p$. Its stable and unstable invariant cylinders intersect each other at four transverse homoclinic orbits as stated in Theorem 1.1. It is well known that every such KAMcurve is a limit of other KAM-curves in $C^{2}$-topology and their invariant stable and unstable cylinders are also close in $C^{2}$-topology on compact sets (under our assumptions of smoothness for $f$ ). Let us fix KAM-curve $\gamma_{1}$ being sufficiently close to $\gamma$. Their $C^{2}$-closeness implies the transverse intersecting of $W^{s}(\gamma)$ and $W^{u}\left(\gamma_{1}\right)$ along four hete- 
roclinic orbits (their traces on $\Sigma$ are transversely intersecting each other in close "almost ellipses"), and vice versa, the same is true for $W^{u}(\gamma)$ and $W^{s}\left(\gamma_{1}\right)$. Thus, there are many heteroclinic connections between different close KAM-curves. The dynamics which follows from this picture is not as completely understood as for the case of homoclinic orbits to a periodic orbit. As was mentioned before, the first contribution here was done in [13. for some model problem. More details can be found in [8, 11]; nevertheless, a complete picture is not understood. Some more details will be presented in the next section.

\section{Homoclinic ORBITS TO SADDLE PERIODIC ORBITS ON $W^{c}$}

As is well known, a sufficiently smooth 2-dimensional symplectic diffeomorphism near its generic elliptic fixed point $O$ (some Birkhoff coefficient in the normal form does not vanish) is a twist map with respect to action-angle variables given by symplectic coordinates of the Birkhoff normal form. In particular, this implies the existence of a positive measure Cantor set of invariant KAM-curves with Diophantine rotation numbers accumulating at $p$ [36]. For our case of such diffeomorphisms we have the restriction of $f$ onto $W^{c}(p)$. Since $W^{c}$ is a smooth normally hyperbolic invariant submanifold for $f$, the Fenichel results [14, 15] that give smooth foliations of $W^{c s}$ and $W^{c u}$ into smooth curves (see Appendix) are applicable. These smooth foliations allow us to define two smooth maps: $F_{u}: W^{c} \rightarrow \Sigma, F_{s}: W^{c} \rightarrow \Sigma$.

Let us fix some invariant KAM-curve $\gamma$ with a Diophantine rotation number. Near such a curve there is a positive measure set of other smooth invariant curves accumulating at $\gamma$ in at least $C^{2}$-topology. Another consequence of the twist condition is that by the Poincaré-Birkhoff theorem for any rational rotation number $l / n$ with incommensurate integers $l, n$ there are at least two $n$-periodic points. Generically, these two periodic orbits are one elliptic and the other hyperbolic. Moreover, if this diffeomorphism satisfies some additional genericity condition (sometimes, this is called the Moser genericity [16]), then stable and unstable separatrices of the hyperbolic orbit intersect transversely along related homoclinic orbits in $W^{c}$. This allows us, in particular, to construct a "fence" made up of stable and unstable separatrices which divorce one invariant curve from another one.

This can be done in the following way. We assume, for simplicity, that $n=2$; thus the related periodic orbits are 2-periodic. Let us fix one hyperbolic 2-periodic point $m_{0}$ and let $m_{1}$ be the second point of this orbit, $m_{1}=f\left(m_{0}\right)$. Suppose that unstable manifold $W^{u}\left(m_{0}\right)$ for the restriction of $f$ on $W^{c}$ transversely intersects the stable manifold of $W^{s}\left(m_{1}\right)$ and that the stable manifold of $W^{s}\left(m_{0}\right)$ transversely intersects the unstable manifold of $W^{u}\left(m_{1}\right)$. In this case, due to the so-called lambda lemma 39, the topological limit of $W^{u}\left(m_{0}\right)$ contains the whole $W^{u}\left(m_{1}\right)$ and vice versa. Thus we get some closed invariant set $\mathfrak{F}_{u}$ made up of these curves and their closures. A similar set $\mathfrak{F}_{s}$ is formed by stable manifolds (see Figure 4).

Take $\mathfrak{F}_{u}$ and for every one of its points consider the related unstable leaf of the unstable foliation in $W^{c u}$. Then map $F_{u}$ transforms set $\mathfrak{F}_{u}$ to the homeomorphic set in $\Sigma$. A similar set in $\Sigma$ is obtained from $\mathfrak{F}_{s}$ using $F_{s}$. Now choose any invariant KAM-curve in $W^{c}$ in a neighborhood $U$ where Theorem 1.1 applies. Choose a sufficiently close invariant KAM-curve $\gamma_{1}$ such that on $\Sigma$ related traces $w^{s}(\gamma), w^{s}\left(\gamma_{1}\right)$ intersect transversely traces $w^{u}(\gamma), w^{u}\left(\gamma_{1}\right)$. Then we have on $\Sigma$ two annuli: $A_{s}$ bounded by $w^{s}(\gamma), w^{s}\left(\gamma_{1}\right)$ and $A_{u}$ bounded by $w^{u}(\gamma), w^{u}\left(\gamma_{1}\right)$. These annuli intersect each other in such a way that each boundary curve of one annulus intersects every boundary curve of another annulus transversely. Since the restriction $f_{c}$ of $f$ on $W^{c}$ is a twist map, then invariant KAMcurves $\gamma, \gamma_{1}$ have different rotation numbers $\rho, \rho_{1}$. Thus there are periodic orbits inside the annulus between $\gamma, \gamma_{1}$ corresponding to some rational $\rho<\rho_{*}<\rho_{1}, \rho_{*}=p / q$. If $f_{c}$ is Moser generic, then half of these periodic orbits are hyperbolic Birkhoff $q$-periodic and 


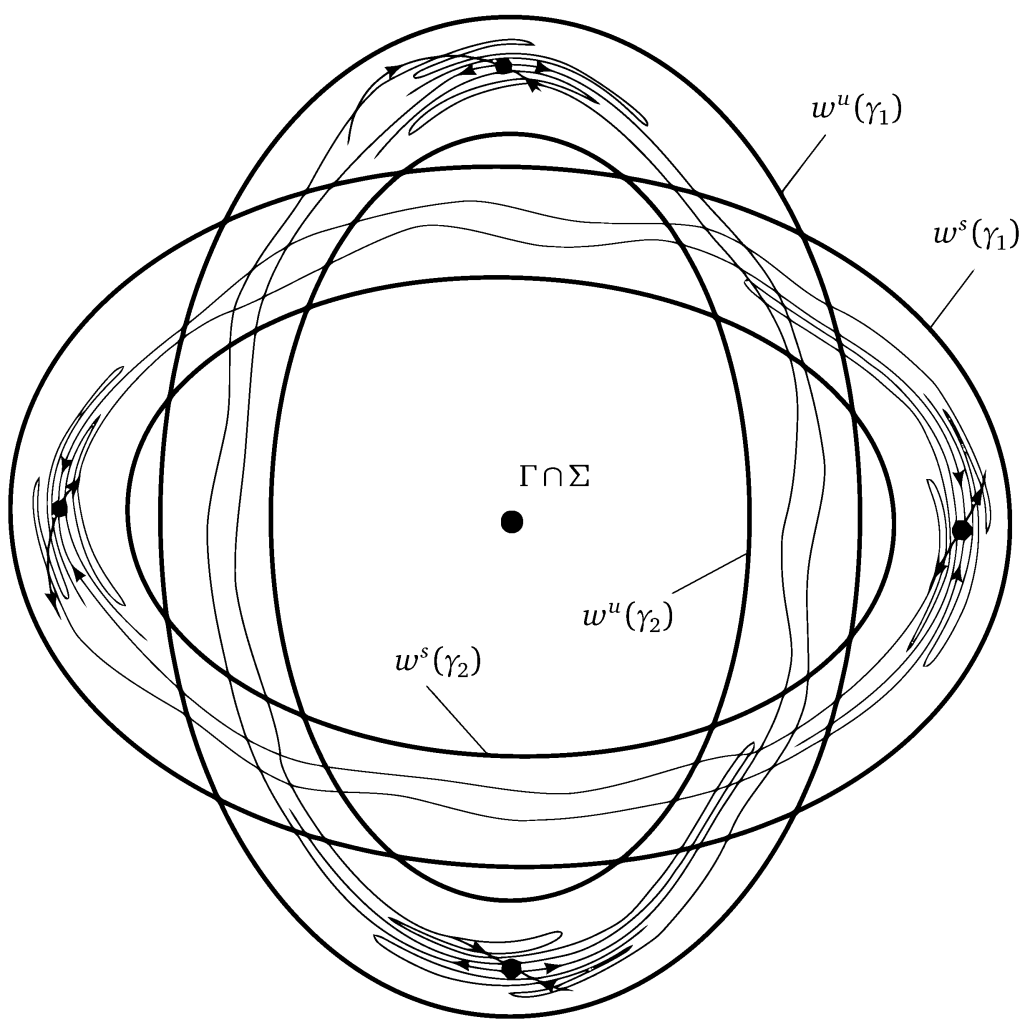

Figure 4. A fence on $\Sigma$ made up of stable and unstable manifolds of a saddle periodic orbit

its stable manifolds form a fence $\mathfrak{F}_{s}$ in $A_{s} \subset \Sigma$. This fence separates $A_{s}$ in the sense that if we take two points on different boundary curves of $A_{s}$, then any path going from one point to another one will cut $\mathfrak{F}_{s}$. The same holds for $\mathfrak{F}_{u}$ in $A_{u}$. This implies

Theorem 5.1. The sets $\mathfrak{F}_{s}, \mathfrak{F}_{u}$ intersect; hence there are Poincaré homoclinic orbits to a saddle hyperbolic periodic orbit on $W^{s}$.

It is clear that in fact there are countably many such Poincaré homoclinic orbits. Of course, it is impossible to assert that they are transverse or tangent since this cannot be caught by such considerations.

\section{Multidimensional extension}

The problem we have studied possesses a multidimensional extension. The tools to get this extension are essentially the same, so we present only the related setup and formulations. In a smooth symplectic manifold $(M, \Omega)$ of dimension $2 n+2$ we consider a symplectic diffeomorphism $f$ that possesses a fixed point $p$ of the elliptic-hyperbolic $(2,2 n)$-type. The latter means this point has the linearization operator with the only pair of complex eigenvalues on the unit circle $\exp [ \pm i \alpha]$ and the remaining $2 n$ eigenvalues are off the unit circle and thus are met either in real pairs $\mu_{k}, \mu_{k}^{-1},\left|\mu_{k}\right|<1$ or in complex quartets $\rho_{m} \exp \left[ \pm i \kappa_{m}\right], \rho_{m}^{-1} \exp \left[ \pm i \kappa_{m}\right], \kappa_{m} \neq 0, \pi$. Here one has $k+2 m=n$. Such a fixed point has locally a smooth 2-dimensional center manifold $W^{c}$ corresponding to the pair $\exp [ \pm i \alpha]$ on which $p$ is an elliptic fixed point, and we assume henceforth that it is of generic elliptic type. Besides the center manifold, through fixed points other smooth 
manifolds pass: $n$-dimensional strong stable $W^{s}$ and strong unstable $W^{u}$ manifolds, as well as $(n+2)$-dimensional center stable $W^{c s}$ and center unstable $W^{c u}$ manifolds.

Analogs of our three Assumptions 13 are:

Assumption 4 (Homoclinic intersection). Manifolds $W^{u}(p)$ and $W^{s}(p)$ have an intersection at some point $q$, generating thus a homoclinic orbit $\Gamma$ to point $p$.

Dimensions of stable $W^{s}(n)$ and center unstable manifold $W^{c u}(n+2)$ are complementary. This allows one to impose

Assumption 5 (Transversality condition). The intersection of manifolds $W^{s}(p)$ and $W^{c u}(p)$ at point $q$ is transverse.

Below we shall show that along the homoclinic orbit $\Gamma$ the sequence of linearized maps generates the linear symplectic scattering map

$$
S: T_{p} W^{c} \rightarrow T_{p} W^{c} .
$$

We assume that this map is generic, which means as above that the foliation into ellipses on the tangent plane $T_{p} W^{c}$ generated by the linearized map $D f_{p}$ has the property that any ellipse $E$ of this foliation satisfies

Assumption 6 (Genericity condition). The intersection $E \cap S(E)$ is transverse and consists of four points.

If these three conditions hold, then the analog of the main theorem is valid.

Theorem 6.1. Let a $C^{r}$-smooth, $r \geq 6$, symplectic diffeomorphism $f$ on a $C^{\infty}$-smooth $2(n+1)$-dimensional symplectic manifold $M$ with an elliptic-hyperbolic fixed point of the type $(2,2 n)$ obey Assumptions 4 6. Then there is a neighborhood $U$ of homoclinic orbit $\Gamma$ such that every closed invariant KAM-curve on $W^{c}$ possesses four transverse homoclinic orbits in $U$.

To prove this theorem we again first study the linearized nonautonomous problem given by the linearization of $f$ on the homoclinic orbit $\Gamma$. Also, in order to avoid possible complications, one assumes in addition that orbit $\Gamma$ leaves from $p$ and enters $p$ along the leading direction in $W^{u}$ and $W^{s}$ (1- or 2-dimensional).

Then, as above, we construct scattering map $S$ acting on $T_{p} W^{c}$ and assuming Assumptions 4 6 to hold we prove the theorem. This proof uses again that the transverse intersection of $W^{c u}$ and $W^{c s}$ near a homoclinic point $q \in \Gamma$ occurs along a 2-dimensional disk $\Sigma$ which belongs to both of them. Hence $(n+1)$-dimensional stable and unstable manifolds of any invariant KAM-curve $\gamma \subset W^{c}$ when continuing by $f$ in $W^{c s}, W^{c s}$, respectively, intersect again $\Sigma$ along closed curves $w^{s}(\gamma), w^{u}(\gamma)$. Genericity Assumption 6 implies this intersection happens transversely at four points through which homoclinic orbits to $\gamma$ pass.

\section{Appendices}

7.1. Straightening invariant manifolds. In some neighborhood of the fixed point the symplectic diffeomorphism under consideration can be in the form (6). In this form the 1-dimensional stable manifold $W^{s}$ is given as a smooth curve tangent to the $x$-axis (at point $p$ ), and the 1-dimensional unstable manifold $W^{u}$ is given as a smooth curve tangent to the $y$-axis. Center stable $W^{c s}$ and center unstable $W^{c u}$ manifolds are given as graphs of the functions $y=F(x, u, v)$ and $x=G(y, u, v)$ being tangent at $p$ to 3-dimensional planes $y=0$ and $x=0$, respectively. Let us first straighten the curves $W^{s}, W^{u}$. 
Lemma 7.1. In linear symplectic space $\left(\mathbb{R}^{4}, \Omega=d x \wedge d y+d u \wedge d v\right)$ let a smooth curve $(x, y(x), u(x), v(x))$ through the point $(0,0,0,0)$ be given such that $y^{\prime}(0)=u^{\prime}(0)=v^{\prime}(0)$ $=0$. Then this curve can be transformed by a symplectic transformation $(x, y, u, v) \rightarrow$ $(\xi, \eta, \nu, \omega)$ into the $\xi$-axis.

There are many such transformations, for instance:

$$
\left\{\begin{array}{l}
\xi=x \\
\eta=y-y(x)-v^{\prime}(x)(u-u(x))+u^{\prime}(x)(v-v(x)) \\
\nu=u-u(x) \\
\omega=v-v(x)
\end{array}\right.
$$

All other transformations in a neighborhood of $p$ we perform holding straight $W^{s}$ and $W^{u}$. At the next step we straighten $W^{c s}$ and $W^{c u}$.

Lemma 7.2. In some neighborhood of point $p$ there exist symplectic coordinates $(x, y$, $u, v), \Omega=d x \wedge d y+d u \wedge d v$ such that submanifolds $W^{c s}, W^{c u}$ in these coordinates become flat; that is, they are given as $y=0, x=0$, respectively.

Proof. In principal, this lemma follows from the related result of the theory of symplectic manifolds (the relative Darboux theorem) [3], but for the reader's convenience, we present a direct proof. We follow the lines of the proof of the Darboux theorem given in [1].

In coordinates (6) $W^{c s}$ is expressed as $y=F(x, u, v)$, where

$$
F(0,0,0)=F_{x}(0,0,0)=F_{u}(0,0,0)=F_{v}(0,0,0)=0 .
$$

Center unstable manifold $W^{c u}$ in the same coordinates is given as $x=G(y, u, v)$,

$$
G(0,0,0)=G_{y}(0,0,0)=G_{u}(0,0,0)=G_{v}(0,0,0)=0 .
$$

Take function $p_{1}=y-F$ as a Hamilton function and consider the related Hamiltonian flow. Since $\dot{x}=1$ then in a neighborhood of $p$ small enough submanifold $x=G(y, u, v)$ is transversal to flow orbits. We take this manifold as a cross-section to the flow. Denote by $q_{1}(x, y, u, v)$ the time needed for the flow orbit through the initial point $\left(x_{0}=\right.$ $\left.G\left(y_{0}, u_{0}, v_{0}\right), y_{0}, u_{0}, v_{0}\right)$ to reach the point $(x, y, u, v)$. Then $q_{1}=0$ for points on the cross-section and $p_{1}=0$ on $W^{c s}$ since it is a level of the Hamiltonian. The Lie derivative of $q_{1}$ w.r.t. the vector field $X_{p_{1}}$ is equal to 1 . Therefore, Hamiltonian vector fields with the Hamilton functions $p_{1}, q_{1}$ commute and are independent in a neighborhood of $p$. Thus, orbits of $\mathbb{R}^{2}$-action generated by these two commuting Hamilton functions give a smooth foliation into 2-dimensional orbits near $p$, and its leaves are transversal to the 2-dimensional submanifold being a joint level of functions $p_{1}$ and $q_{1}$. Next we take joint level $p_{1}=q_{1}=0$ that is just locally $W^{c}$. We introduce any local symplectic w.r.t. the restriction of 2 -form $\Omega$ on $W^{c}$ coordinates $\left(p_{2}, q_{2}\right)$ near $p$. These coordinates are extended onto a neighborhood of $W^{c}$ setting $\left(p_{2}, q_{2}\right)$ constant along the whole 2-dimensional orbit of the action through the point on $W^{c}$ with coordinates $\left(p_{2}, q_{2}\right)$ on it.

Remark 7.3. If $W^{s}$ and $W^{u}$ were previously made straight, then one has

$$
F(x, 0,0) \equiv 0, \quad G(y, 0,0) \equiv 0,
$$

and straightening $W^{c s}, W^{c u}$ preserves $W^{s}, W^{u}$ straight.

7.2. Normal form near 1-elliptic fixed point. Here we shall derive the normal form up to the terms of third order for a smooth symplectic 4-dim diffeomorphism $f$ in neighborhood $V$ of its fixed 1-elliptic point $p$. Without loss of generality one may assume $\alpha \in(0, \pi)$. 
Proposition 7.4. In some neighborhood $V$ of fixed 1-elliptic point p there exist symplectic coordinates $(x, y, u, v), \Omega=d x \wedge d y+d u \wedge d v$ such that diffeomorphism $f$ has the following form in these coordinates:

$$
\left\{\begin{aligned}
x_{n+1}= & \mu x_{n}\left(1+a x_{n} y_{n}+b\left(u_{n}^{2}+v_{n}^{2}\right)+\mathcal{O}_{3}\right), \\
y_{n+1}= & \mu^{-1} y_{n}\left(1-a x y-b\left(u_{n}^{2}+v_{n}^{2}\right)+\mathcal{O}_{3}\right), \\
u_{n+1}= & u_{n} \cos \left(\alpha+\nu\left(u_{n}^{2}+v_{n}^{2}\right)\right)-v_{n} \sin \left(\alpha+\nu\left(u_{n}^{2}+v_{n}^{2}\right)\right) \\
& -\kappa x_{n} y_{n}\left(u_{n} \sin \alpha+v_{n} \cos \alpha\right)+\varphi\left(x_{n}, y_{n}, u_{n}, v_{n}\right), \\
v_{n+1}= & u_{n} \sin \left(\alpha+\nu\left(u_{n}^{2}+v_{n}^{2}\right)\right)+v_{n} \cos \left(\alpha+\nu\left(u_{n}^{2}+v_{n}^{2}\right)\right) \\
& +\kappa x_{n} y_{n}\left(u_{n} \cos \alpha-v_{n} \sin \alpha\right)+\psi\left(x_{n}, y_{n}, u_{n}, v_{n}\right),
\end{aligned}\right.
$$

where $\nu \neq 0$, functions $\varphi, \psi$ are of the fourth order and higher at the origin, $\mathcal{O}_{3}$ means terms of third order and higher at the origin. In these coordinates manifolds $W^{s}, W^{u}$ coincide with the $x$-axis, $y$-axis, respectively; that is, the following identities hold:

$$
\varphi(x, 0,0,0)=\varphi(0, y, 0,0)=\psi(x, 0,0,0)=\psi(0, y, 0,0) \equiv 0 .
$$

Proof. At the first step we straighten manifolds $W^{c s}, W^{c u}$ in the neighborhood of $p$ (see Appendix). As a result, the first two relations in (6) are transformed into the form

$$
x_{n+1}=x_{n}(\mu+\ldots), \quad y_{n+1}=y_{n}\left(\mu^{-1}+\ldots\right) .
$$

Next we apply the standard normal form method for symplectic maps (see for instance [3]). We shall use such symplectic coordinate transformations which hold that $W^{c s}, W^{c u}$ be straightened. Next we use complex coordinates instead of $u, v$ in order to diagonalize the linear part of the third and fourth relations. Monomials of the second order and those of third order other than resonance monomials can be killed.

Resonance relations for the set of eigenvalues $\left(\mu, \mu^{-1}, e^{i \alpha}, e^{-i \alpha}\right)$ and integer vectors $\left(m_{1}, m_{2}, n_{1}, n_{2}\right)$ have the form

$$
\begin{array}{ll}
\mu^{m_{1}-m_{2}-1} e^{i \alpha\left(n_{1}-n_{2}\right)}=1, & \mu^{m_{1}-m_{2}+1} e^{i \alpha\left(n_{1}-n_{2}\right)}=1, \\
\mu^{m_{1}-m_{2}} e^{i \alpha\left(n_{1}-n_{2}-1\right)}=1, & \mu^{m_{1}-m_{2}} e^{i \alpha\left(n_{1}-n_{2}+1\right)}=1 .
\end{array}
$$

These relations can be rewritten in the following way:

$$
\begin{array}{lll}
m_{1}=m_{2}+1, & \alpha\left(n_{1}-n_{2}\right)=2 \pi k, & k \in \mathbb{Z} ; \\
m_{2}=m_{1}+1, & \alpha\left(n_{1}-n_{2}\right)=2 \pi k, & k \in \mathbb{Z} ; \\
m_{1}=m_{2}, & \alpha\left(n_{1}-n_{2}-1\right)=2 \pi k, & k \in \mathbb{Z} ; \\
m_{1}=m_{2}, & \alpha\left(n_{1}-n_{2}+1\right)=2 \pi k, & k \in \mathbb{Z} .
\end{array}
$$

From these relations for integer vectors $\left(m_{1}, m_{2}, n_{1}, n_{2}\right)$ such that

$$
|m|+|n|=m_{1}+m_{2}+n_{1}+n_{2}=2,3
$$

we derive that if $|m|+|n|=2$, then resonance relations (16) and (17) are absent, and resonance relations (18) and (19) are the same as for the case of 2-dimensional elliptic point $\alpha=2 \pi / 3$. Thus, according to our assumptions (that $p$ is a 1-elliptic fixed point of generic type for $\left.f\right|_{W^{c}}$ ) resonant monomials of second order can be removed. If $|m|+|n|=3$, then for resonance relations (16) we get resonant monomials $x^{2} y$, corresponding to the 2 -dimensional saddle point of symplectic diffeomorphism, and $x\left(u^{2}+v^{2}\right)$. For relations (17) we get $x y^{2}$ and $y\left(u^{2}+v^{2}\right)$. Under an assumption that strong resonances are absent in the system, relations (18) and (19) show resonant monomials $x y u, x y v, u\left(u^{2}+v^{2}\right)$ and $v\left(u^{2}+v^{2}\right)$ cannot be eliminated. The last two present in the normal form for a diffeomorphism in a neighborhood of an elliptic point. Taking into account that the transformation to the normal form should be symplectic we get (15). 
7.3. Invariant foliations and their smoothness. In this subsection we verify the conditions from [14, 15] which guarantee the existence and smoothness of stable and unstable invariant foliations within manifolds $W^{c s}, W^{c u}$, respectively. In particular, these conditions imply the existence of stable and unstable smooth invariant manifolds for KAM-curves on the center manifold $W^{c}$. Homoclinic orbits to KAM-curves belong to the intersection of these manifolds. Note that this fact does not follow immediately from the Hirsch-Pugh-Shub theorem [21], and we use the theory developed by Fenichel [14, 15]. Let us recall the definition of a weakly overflowing invariant set according to 14 .

Definition 7.5 (N. Fenichel, 1974). Let $U$ and $V$ be open subsets of some $C^{l}$-manifold $M_{1}, 1 \leq l \leq \infty$, and let $F: U \rightarrow V$ be a $C^{l}$-diffeomorphism. A set $\Lambda \subset U$ is called weakly overflowing invariant (under $F$ ) if $\Lambda \subset F(\Lambda)$.

Let $T F: T U \rightarrow T V$ be the map induced by $F$ on tangent spaces. A subbundle $\left.E \subset T M_{1}\right|_{\Lambda}$ is called weakly overflowing invariant if $E \subset T F(E)$.

Let us choose any invariant KAM-curve on $W^{c}$ in a sufficiently small neighborhood of $p$. Then the closure of subsets in $W^{c}$ bounded by this KAM-curve is a weakly overflowing invariant set under diffeomorphism $f$. Denote this compact set as $\Lambda$. Here we assume $M_{1}=W^{c u}$ and consider $\left.f\right|_{M_{1}}$. To apply expanding family theorems one needs to show that there exists weakly overflowing invariant subbundle $\left.E \subset T M_{1}\right|_{\Lambda}$. It will be proved using the contraction mapping principle. Recall that locally near $p$ manifolds $W^{c u}, W^{c s}$ are straightened (i.e. $x \equiv 0$ on $W^{c u}$ and $y \equiv 0$ on $\left.W^{c s}\right)$ and $(y, u, v)$ are coordinates on it. Then the restriction $f$ on $W^{c u}$ has the following form:

$$
\left\{\begin{array}{l}
y_{n+1}=\mu^{-1} y_{n}+h\left(y_{n}, u_{n}, v_{n}\right) y_{n}, \\
u_{n+1}=u_{n} \cos \alpha-v_{n} \sin \alpha+g_{1}\left(y_{n}, u_{n}, v_{n}\right), \\
v_{n+1}=u_{n} \sin \alpha+v_{n} \cos \alpha+g_{2}\left(y_{n}, u_{n}, v_{n}\right),
\end{array}\right.
$$

where functions $g_{1}, g_{2}$ are of second order in $(y, u, v), h$ is a first-order function. Now let us change variables $\left(y_{n}, u_{n}, v_{n}\right) \rightarrow\left(\widehat{y}_{n}, \widehat{u}_{n}, \widehat{v}_{n}\right)$ :

$$
\left\{\begin{array}{c}
y_{n}=\widehat{y}_{n}, \\
\left(\begin{array}{l}
u_{n} \\
v_{n}
\end{array}\right)=R_{n \alpha}\left(\begin{array}{l}
\widehat{u}_{n} \\
\widehat{v}_{n}
\end{array}\right) .
\end{array}\right.
$$

In new coordinates the diffeomorphism will have the form (we keep old notation for variables):

$$
\left\{\begin{array}{l}
y_{n+1}=\mu^{-1} y_{n}+h\left(y_{n}, u_{n}, v_{n}\right) y_{n} \\
u_{n+1}=u_{n}+g_{1}\left(y_{n}, u_{n}, v_{n}\right) \\
v_{n+1}=v_{n}+g_{2}\left(y_{n}, u_{n}, v_{n}\right)
\end{array}\right.
$$

At any point $m \in \Lambda$ differential $D f$ has the following representation (recall $\Lambda \subset W^{c}$ is given as $y \equiv 0)$ :

$$
D f_{m}=\left(\begin{array}{ccc}
\mu^{-1}+h(m) & 0 & 0 \\
g_{1_{y}}(m) & 1+g_{1_{u}}(m) & g_{1_{v}}(m) \\
g_{2_{y}}(m) & g_{2_{u}}(m) & 1+g_{2_{v}}(m)
\end{array}\right) .
$$

Since we work in one coordinate chart $(y, u, v)$, we will denote $\left(\eta, \chi_{1}, \chi_{2}\right)$ as coordinates in the tangent space to a point $m$. Now consider any orbit $\left(\ldots, m_{n}, m_{n+1}, \ldots\right)$ of $\left.f\right|_{W^{c}}$, $m=m_{0}$, which belongs to $\Lambda$ (that is to $W^{c}$ ). At each point of this orbit choose in $T_{m_{n}} M_{1}$ a straight line through the origin in the tangent space being transversal to plane $\eta=0$. Such a straight line can be given parametrically: $\left(\eta, p_{n} \eta, q_{n} \eta\right), \eta \in \mathbb{R}$, functions $p_{n}$ and 
$q_{n}$ smoothly depend on $m$ in $\Lambda$. Differential $D f_{m}$ transforms this line to another line in $T_{f(m)} M_{1}$ :

$$
\begin{aligned}
\bar{\eta} & =\left(\mu^{-1}+h\left(m_{n}\right)\right) \eta \\
\bar{p}_{n+1} & =\frac{g_{1_{y}}\left(m_{n}\right)+\left(1+g_{1_{u}}\left(m_{n}\right)\right) p_{n}+g_{1_{v}}\left(m_{n}\right) q_{n}}{\mu^{-1}+h\left(m_{n}\right)}, \\
\bar{q}_{n+1} & =\frac{g_{2_{y}}\left(m_{n}\right)+g_{2_{u}}\left(m_{n}\right) p_{n}+\left(1+g_{2_{v}}\left(m_{n}\right)\right) q_{n}}{\mu^{-1}+h\left(m_{n}\right)} .
\end{aligned}
$$

The r.h.s. of the second and third relations define an operator in the Banach space of uniformly bounded sequences $\zeta=\left\{\left(p_{n}, q_{n}\right)\right\}$ with norm $\|\zeta\|=\sup _{n \in \mathbb{Z}}\left(\left|p_{n}\right|,\left|q_{n}\right|\right)$. Indeed, this operator will transform a uniformly bounded sequence $\zeta$ to the bounded one, as all derivatives $\left(g_{i}\right)_{y}^{\prime},\left(g_{i}\right)_{u}^{\prime},\left(g_{i}\right)_{v}^{\prime}, i=1,2$, calculated at $m_{n}$, are small (functions $g_{i}, i=1,2$, are of second order at zero):

$$
\begin{aligned}
\left|\bar{p}_{n+1}\right| & \leq \frac{\left|g_{1_{y}}\left(m_{n}\right)\right|+\left|1+g_{1_{u}}\left(m_{n}\right)\right| \cdot\|\zeta\|+\left|g_{1_{v}}\left(m_{n}\right)\right| \cdot\|\zeta\|}{\left|\mu^{-1}+h\left(m_{n}\right)\right|}, \\
\left|\bar{q}_{n+1}\right| & \leq \frac{\left|g_{2_{y}}\left(m_{n}\right)\right|+\left|g_{2_{u}}\left(m_{n}\right)\right| \cdot\|\zeta\|+\left|1+g_{2_{v}}\left(m_{n}\right)\right| \cdot\|\zeta\|}{\left|\mu^{-1}+h\left(m_{n}\right)\right|} .
\end{aligned}
$$

To prove the operator is contracting, consider the following inequalities:

$$
\begin{gathered}
\left|\bar{p}_{n+1}^{1}-\bar{p}_{n+1}^{2}\right| \leq \frac{\left|1+g_{1_{u}}\left(m_{n}\right)\right| \cdot\left|p_{n}^{1}-p_{n}^{2}\right|+\left|g_{1_{v}}\left(m_{n}\right)\right| \cdot\left|q_{n}^{1}-q_{n}^{2}\right|}{\left|\mu^{-1}+h\left(m_{n}\right)\right|} \\
\leq \frac{\left(\left|1+g_{1_{u}}\left(m_{n}\right)\right|+\left|g_{1_{v}}\left(m_{n}\right)\right|\right)\left\|\zeta^{1}-\zeta^{2}\right\|}{\left|\mu^{-1}+h\left(m_{n}\right)\right|}, \\
\left|\bar{q}_{n+1}^{1}-\bar{q}_{n+1}^{2}\right| \leq \frac{\left|g_{2_{u}}\left(m_{n}\right)\right| \cdot\left|p_{n}^{1}-p_{n}^{2}\right|+\left|1+g_{2_{v}}\left(m_{n}\right)\right| \cdot\left|q_{n}^{1}-q_{n}^{2}\right|}{\left|\mu^{-1}+h\left(m_{n}\right)\right|} \\
\leq \frac{\left(\left|g_{2_{u}}\left(m_{n}\right)\right|+\left|1+g_{2_{v}}\left(m_{n}\right)\right|\right)\left\|\zeta^{1}-\zeta^{1}\right\|}{\left|\mu^{-1}+h\left(m_{n}\right)\right|} .
\end{gathered}
$$

Quantities such as

$$
\frac{\left|1+g_{1_{u}}\left(m_{n}\right)\right|+\left|g_{1_{v}}\left(m_{n}\right)\right|}{e f t\left|\mu^{-1}+h\left(m_{n}\right)\right|}
$$

are less than 1 uniformly in $n$ if we are working in a neighborhood of $p$ small enough so that the operator is contracting. According to the contraction mapping principle there exists a unique fixed point of the operator, namely some sequence $\left\{\left(p_{n}^{*}, q_{n}^{*}\right)\right\}$. This sequence (as the point in the related Banach space) depends continuously on $m \in \Lambda$. The straight lines corresponding to this sequence, as $m$ varies along $\Lambda$, form weakly overflowing invariant subbundle $\left.E \subset T M_{1}\right|_{\Lambda}$.

Next we choose a vector bundle $\left.N \subset T M_{1}\right|_{\Lambda}$, complementary to $E$, that is, $\left.T M_{1}\right|_{\Lambda}=$ $=E \oplus N$. We set $N=T \Lambda$. According to [14] for $m \in \Lambda$, any $v_{0} \in E$ and $W_{0} \in N$ let

$$
v_{-k}=D f^{-k}(m) v_{0}, \quad w_{-k}=\pi^{N} D f^{-k}(m) w_{0},
$$

where $\pi^{N}$ is a projection to $N$ (note that in our case $N$ is invariant under $D f$, so one can just let $\left.w_{-k}=D f^{-k}(m) w_{0}\right)$. Let us also define two numbers:

$$
\begin{aligned}
\alpha^{*}(m) & =\inf _{\alpha>0}\left\{\left|v_{-k}\right| / \alpha^{k} \rightarrow 0 \text { as } k \rightarrow \infty \forall v_{0} \in E\right\}, \\
\rho^{*}(m) & =\inf _{\rho>0}\left\{\left|v_{-k}\right| /\left|w_{-k}\right| / \rho^{k} \rightarrow 0 \text { as } k \rightarrow \infty \forall v_{0} \in E, w_{0} \in N\right\} .
\end{aligned}
$$


The number $\alpha^{*}(m)$ is an asymptotic measure of the growth of vectors in $E$ under the action of $D f^{-1}$, and $\rho^{*}(m)$ is an asymptotic measure of the ratio of the growth of vectors in $E$ to the growth of vectors in $N$ under the action of $D f^{-1}$.

We recall one more definition and formulate th expanding family theorem 14 for the reader's convenience.

Definition 7.6 (N. Fenichel, 1974). The pair $(\Lambda, E)$ is called an invariant set with expanding structure for $f$ if $\Lambda$ is a compact and weakly overflowing invariant, $E$ is a weakly overflowing invariant, and $\alpha^{*}(m)<1, \rho^{*}(m)<1$ for all $m \in \Lambda$.

Theorem 7.7 (Expanding Family Theorem, N. Fenichel, 1974). Let $M_{1}$ be a $C^{l}$-manifold, $1 \leq l \leq \infty$, and let $F: M_{1} \rightarrow M_{1}$ be a $C^{l}$-diffeomorphism. Let $(\Lambda, E)$ be an invariant set with expanding structure. Then there is a family of $C^{l}$-manifolds $W^{E}(m), m \in \Lambda$, invariant in the sense that

$$
F^{-1}\left(W^{E}(m)\right)=W^{E}\left(F^{-1}(m)\right) .
$$

The manifold $W^{E}(m)$ is $C^{l}$-diffeomorphic to the fiber $E_{m}$ and is tangent to $E_{m}$ at $m$.

Let us show that in our case $\alpha^{*}(m)<1, \rho^{*}(m)<1$ for any $m \subset \Lambda$. Taking any $v_{0} \in E$, it has the coordinate representation $v_{0}=\left(\eta_{0}, p_{0}^{*} \eta_{0}, q_{0}^{*} \eta_{0}\right)$. Vector $v_{-k}$ will have the representation $v_{-k}=\left(\eta_{-k}, p_{-k}^{*} \eta_{-k}, q_{-k}^{*} \eta_{-k}\right)$. Taking into account the first equality from (21) one gets:

$$
\begin{aligned}
& \eta_{-1}=\frac{\mu \eta_{0}}{1+\mu h\left(m_{-1}\right)}, \\
& \eta_{-2}=\frac{\mu \eta_{-1}}{1+\mu h\left(m_{-2}\right)}=\frac{\mu^{2} \eta_{0}}{\left(1+\mu h\left(m_{-1}\right)\right)\left(1+\mu h\left(m_{-2}\right)\right)}, \\
& \ldots \ldots \ldots \ldots \ldots \ldots \ldots \ldots \ldots \ldots \ldots \ldots \ldots \ldots \ldots \ldots \ldots \ldots \ldots \ldots \ldots \ldots \ldots \ldots \ldots \ldots \ldots \\
& \eta_{-k}=\frac{\mu^{k} \eta_{0}}{\left(1+\mu h\left(m_{-1}\right)\right) \ldots\left(1+\mu h\left(m_{-k}\right)\right)}
\end{aligned}
$$

Now consider the ratio

$$
\frac{\left|v_{-k}\right|}{\alpha^{k}}=\frac{\left|\eta_{-k}\right| \sqrt{1+\left(p_{-k}^{*}\right)^{2}+\left(q_{-k}^{*}\right)^{2}}}{\alpha^{k}}=\frac{\mu^{k}\left|\eta_{0}\right| \sqrt{1+\left(p_{-k}^{*}\right)^{2}+\left(q_{-k}^{*}\right)^{2}}}{\alpha^{k}\left|\left(1+\mu h\left(m_{-1}\right)\right) \ldots\left(1+\mu h\left(m_{-k}\right)\right)\right|} .
$$

Quantity $\left|\eta_{0}\right| \sqrt{1+\left(p_{-k}^{*}\right)^{2}+\left(q_{-k}^{*}\right)^{2}}$ is bounded. Function $h$ is of the first order, and $m_{n}$ lies in a small neighborhood of fixed point $p$. Let us define $\delta_{1}=\sup _{n \in \mathbb{Z}}\left|h\left(m_{n}\right)\right|$. This value is of order of size (radius) of the neighborhood and hence is small enough. The following estimates are valid:

$$
\frac{\mu^{k}\left|\eta_{0}\right| \sqrt{1+\left(p_{-k}^{*}\right)^{2}+\left(q_{-k}^{*}\right)^{2}}}{\alpha^{k}\left(1+\mu \delta_{1}\right)^{k}} \leq \frac{\left|v_{-k}\right|}{\alpha^{k}} \leq \frac{\mu^{k}\left|\eta_{0}\right| \sqrt{1+\left(p_{-k}^{*}\right)^{2}+\left(q_{-k}^{*}\right)^{2}}}{\alpha^{k}\left(1-\mu \delta_{1}\right)^{k}} .
$$

The quantity in the r.h.s. of inequality (22) tends to zero as $k \rightarrow \infty$ if $\mu /\left(\alpha\left(1-\mu \delta_{1}\right)\right)<1$, that is, $\alpha>\mu /\left(1-\mu \delta_{1}\right)$. On the other hand, the quantity in the l.h. s. of inequality (22) tends to zero as $k \rightarrow \infty$ if $\alpha>\mu /\left(1+\mu \delta_{1}\right)$. Thus, we get

$$
\alpha^{*}\left(m_{0}\right) \in\left[\frac{\mu}{1+\mu \delta_{1}}, \frac{\mu}{1-\mu \delta_{1}}\right]<1 .
$$

The inverse map for $D f_{m_{n}}$ has the following representation:

$$
D f_{m_{n}}^{-1}=\left(\begin{array}{ccc}
\frac{\mu}{1+\mu h\left(m_{n}\right)} & 0 & 0 \\
\cdots & 1+l_{1}\left(m_{n}\right) & l_{2}\left(m_{n}\right) \\
\cdots & l_{3}\left(m_{n}\right) & 1+l_{4}\left(m_{n}\right)
\end{array}\right)
$$


where dots, $l_{1}, l_{2}, l_{3}$, and $l_{4}$ are of at least first-order functions. Taking any $w_{0} \in N$, it has the form $w_{0}=\left(0, c_{0}, d_{0}\right)$, and let us consider the $k$-th iteration of $w_{0}$ under $D F^{-1}$ : $w_{-k}=\left(0, c_{-k}, d_{-k}\right)$. From (23) one gets that coordinates of $w_{-k}$ change as follows:

$$
\begin{aligned}
& c_{-k}=\left(1+l_{1}\left(m_{-k+1}\right)\right) c_{-k+1}+l_{2}\left(m_{-k+1}\right) d_{-k+1}, \\
& d_{-k}=l_{3}\left(m_{-k+1}\right) c_{-k+1}+\left(1+l_{4}\left(m_{-k+1}\right)\right) d_{-k+1} .
\end{aligned}
$$

Denote

$$
\delta_{2}=\sup _{i} \sup _{n \in \mathbb{Z}}\left|l_{i}\left(m_{n}\right)\right|, \quad i=\overline{1,4} .
$$

This quantity is also small enough and of the order of the size of the neighborhood. The next estimates are valid:

$$
\begin{aligned}
& \left|c_{-k}\right| \leq\left(1+\delta_{2}\right)\left|c_{-k+1}\right|+\delta_{2}\left|d_{-k+1}\right|=\left|c_{-k+1}\right|+\delta_{2}\left(\left|c_{-k+1}\right|+\left|d_{-k+1}\right|\right), \\
& \left|d_{-k}\right| \leq \delta_{2}\left|c_{-k+1}\right|+\left(1+\delta_{2}\right)\left|d_{-k+1}\right|=\left|d_{-k+1}\right|+\delta_{2}\left(\left|c_{-k+1}\right|+\left|d_{-k+1}\right|\right),
\end{aligned}
$$

and so

$$
\begin{aligned}
\left|c_{-k}\right|+\left|d_{-k}\right| & \leq\left|c_{-k+1}\right|+\left|d_{-k+1}\right|+2 \delta_{2}\left(\left|c_{-k+1}\right|+\left|d_{-k+1}\right|\right) \\
& =\left(\left|c_{-k+1}\right|+\left|d_{-k+1}\right|\right)\left(1+2 \delta_{2}\right) \\
& \leq\left(\left|c_{-k+2}\right|+\left|d_{-k+2}\right|\right)\left(1+2 \delta_{2}\right)^{2} \leq \ldots \leq\left(\left|c_{0}\right|+\left|d_{0}\right|\right)\left(1+2 \delta_{2}\right)^{k} .
\end{aligned}
$$

Then one gets

$$
\begin{aligned}
& \sqrt{\left|c_{-k}\right|^{2}+\left|d_{-k}\right|^{2}} \leq \sqrt{2\left(\max \left\{\left|c_{-k}\right|,\left|d_{-k}\right|\right\}\right)^{2}} \leq \sqrt{2} \max \left\{\left|c_{-k}\right|,\left|d_{-k}\right|\right\} \\
& \leq \sqrt{2}\left(\left|c_{-k}\right|+\left|d_{-k}\right|\right) \leq \sqrt{2}\left(\left|c_{0}\right|+\left|d_{0}\right|\right)\left(1+2 \delta_{2}\right)^{k} .
\end{aligned}
$$

On the other side:

$$
\begin{aligned}
& \left|c_{-k}\right| \geq\left(1-\delta_{2}\right)\left|c_{-k+1}\right|-\delta_{2}\left|d_{-k+1}\right|=\left|c_{-k+1}\right|-\delta_{2}\left(\left|c_{-k+1}\right|+\left|d_{-k+1}\right|\right), \\
& \left|d_{-k}\right| \geq\left(1-\delta_{2}\right)\left|d_{-k+1}\right|-\delta_{2}\left|c_{-k+1}\right|=\left|d_{-k+1}\right|-\delta_{2}\left(\left|c_{-k+1}\right|+\left|d_{-k+1}\right|\right),
\end{aligned}
$$

and, consequently,

$$
\begin{aligned}
\left|c_{-k}\right|+\left|d_{-k}\right| & \geq\left|c_{-k+1}\right|+\left|d_{-k+1}\right|-2 \delta_{2}\left(\left|c_{-k+1}\right|+\left|d_{-k+1}\right|\right) \\
& =\left(\left|c_{-k+1}\right|+\left|d_{-k+1}\right|\right)\left(1-2 \delta_{2}\right) \\
& \geq\left(\left|c_{-k+2}\right|+\left|d_{-k+2}\right|\right)\left(1-2 \delta_{2}\right)^{2} \geq \ldots \geq\left(\left|c_{0}\right|+\left|d_{0}\right|\right)\left(1-2 \delta_{2}\right)^{k} .
\end{aligned}
$$

The following inequalities are valid:

$$
\left(\left|c_{0}\right|+\left|d_{0}\right|\right)\left(1-2 \delta_{2}\right)^{k} \leq\left|c_{-k}\right|+\left|d_{-k}\right| \leq 2 \max \left\{\left|c_{-k}\right|,\left|d_{-k}\right|\right\},
$$

so

$$
\max \left\{\left|c_{-k}\right|,\left|d_{-k}\right|\right\} \geq \frac{\left|c_{0}\right|+\left|d_{0}\right|}{2}\left(1-2 \delta_{2}\right)^{k}
$$

and

$$
\sqrt{\left|c_{-k}\right|^{2}+\left|d_{-k}\right|^{2}} \geq \sqrt{\left(\max \left\{\left|c_{-k}\right|,\left|d_{-k}\right|\right\}\right)^{2}}=\max \left\{\left|c_{-k}\right|,\left|d_{-k}\right|\right\} \geq \frac{\left|c_{0}\right|+\left|d_{0}\right|}{2}\left(1+2 \delta_{2}\right)^{k} .
$$

Now let us evaluate

$$
\frac{\left|v_{-k}\right|}{\left|w_{-k}\right| \rho^{k}}=\frac{\mu^{k}\left|\eta_{0}\right| \sqrt{1+\left(p_{-k}^{*}\right)^{2}+\left(q_{-k}^{*}\right)^{2}}}{\rho^{k}\left|\left(1+\mu h\left(m_{-1}\right)\right) \ldots\left(1+\mu h\left(m_{-k}\right)\right)\right| \sqrt{\left|c_{-k}\right|^{2}+\left|d_{-k}\right|^{2}}} .
$$


The next estimates are valid:

$$
\begin{aligned}
& \frac{\left|v_{-k}\right|}{\left|w_{-k}\right| \rho^{k}} \geq \frac{\mu^{k}\left|\eta_{0}\right| \sqrt{1+\left(p_{-k}^{*}\right)^{2}+\left(q_{-k}^{*}\right)^{2}}}{\rho^{k}\left(1+\mu \delta_{1}\right)^{k} \sqrt{2}\left(\left|c_{0}\right|+\left|d_{0}\right|\right)\left(1+2 \delta_{2}\right)^{k}}, \\
& \frac{\left|v_{-k}\right|}{\left|w_{-k}\right| \rho^{k}} \leq \frac{2 \mu^{k}\left|\eta_{0}\right| \sqrt{1+\left(p_{-k}^{*}\right)^{2}+\left(q_{-k}^{*}\right)^{2}}}{\rho^{k}\left(1-\mu \delta_{1}\right)^{k}\left(\left|c_{0}\right|+\left|d_{0}\right|\right)\left(1-2 \delta_{2}\right)^{k}} .
\end{aligned}
$$

The expression in the r.h.s. of the first inequality in (24) tends to zero as $k$ tends to $\infty$ if $\rho>\frac{\mu}{\left(1+\mu \delta_{1}\right)\left(1+2 \delta_{2}\right)}$, while the expression in the r.h.s. of the second inequality in (24) tends to zero as $k$ tends to $\infty$ if $\rho>\frac{\mu}{\left(1-\mu \delta_{1}\right)\left(1-2 \delta_{2}\right)}$. Thus, we get

$$
\rho^{*}\left(m_{0}\right) \in\left[\frac{\mu}{\left(1+\mu \delta_{1}\right)\left(1+2 \delta_{2}\right)}, \frac{\mu}{\left(1-\mu \delta_{1}\right)\left(1-2 \delta_{2}\right)}\right]<1 .
$$

Then the expanding family theorem holds, and for each point $m \in \Lambda$ there exists a 1dimensional manifold (a curve) in $M_{1}$ being tangent to the corresponding layer in $E$. The collection of these curves is invariant under $f^{-1}$. In particular, collecting these manifolds for points of an invariant KAM-curve defines its unstable manifold.

Now we want to have smoothness properties for the expanding foliation obtained. Let us apply the smoothness theorem for invariant sets with expanding structure to prove that these manifolds smoothly depend on $m_{0}$ [15]. For this purpose we define the quantity $\tau^{*}$ according to [15]:

$$
\begin{aligned}
\tau^{*}\left(m_{0}\right)=\inf \left\{\tau:\left[\left|v_{-k}\right| /\left|w_{-k}\right|\right]^{\tau}\left|\xi_{-k}\right| \rightarrow 0\right. \text { as } & k \rightarrow \inf \\
& \left.\forall v_{0} \in E, w_{0} \in N, \xi_{0} \in T_{m_{0}} \Lambda=N\right\} .
\end{aligned}
$$

Theorem 7.8 (Smooth Invariant Bundle Theorem, N. Fenichel, 1977). Let $U$ and $V$ be open subsets of a $C^{l}$-manifold $M_{1}$, and let $F: U \rightarrow V$ be a $C^{l}$-diffeomorphism, $2 \leq l \leq \infty$. Let $\Lambda$ be a compact, properly embedded $C^{l}$-manifold with boundary, overflowing invariant under $F$. Let $(\Lambda, E)$ be an invariant set with expanding structure. If $1 \leq l^{\prime} \leq l-1$ and $\tau^{*}(m)<1 / l^{\prime}$ for all $m \in \Lambda$, then $E$ is a $C^{l^{\prime}}$-smooth vector bundle.

Taking into account estimates found before, one gets for diffeomorphism (20) and any vector $\xi_{0}=\left(0, \bar{c}_{0}, \bar{d}_{0}\right) \in N, \xi_{-k}=\left(0, \bar{c}_{-k}, \bar{d}_{-k}\right)$ that the following inequality is valid:

$$
\begin{aligned}
\frac{\left|v_{-k}\right|^{\tau}}{\left|w_{-k}\right|^{\tau}}\left|\xi_{-k}\right| & =\left(\frac{\mu^{k}\left|\eta_{0}\right| \sqrt{1+\left(p_{-k}^{*}\right)^{2}+\left(q_{-k}^{*}\right)^{2}}}{\left|\left(1+\mu h\left(m_{-1}\right)\right) \ldots\left(1+\mu h\left(m_{-k}\right)\right)\right| \sqrt{\left|c_{-k}\right|^{2}+\left|d_{-k}\right|^{2}}}\right)^{\tau} \sqrt{\left|\bar{c}_{-k}\right|^{2}+\left|\bar{d}_{-k}\right|^{2}} \\
& \leq \frac{\mu^{\tau k} C_{1}^{\tau}}{\left(1-\mu \delta_{1}\right)^{\tau k}\left(1-2 \delta_{2}\right)^{\tau k}} \sqrt{2}\left(\left|\bar{c}_{0}\right|+\left|\bar{d}_{0}\right|\right)\left(1+2 \delta_{2}\right)^{k} .
\end{aligned}
$$

Here $C_{1}$ is constant, which can be easily calculated. The r.h.s. of (25) tends to zero as $k \rightarrow \infty$ if

$$
\tau \geq \tau_{1}=\frac{\ln \left(1+2 \delta_{2}\right)}{\ln \left[\left(1-\mu \delta_{1}\right)\left(1-2 \delta_{2}\right)\right]-\ln \mu}>0 .
$$

Quantity $\tau_{1}$ is small enough of order $\delta_{2}$. On the other hand,

$$
\frac{\left|v_{-k}\right|^{\tau}}{\left|w_{-k}\right|^{\tau}}\left|\xi_{-k}\right| \geq \frac{\mu^{\tau k} C_{2}^{\tau}}{\left(1+\mu \delta_{1}\right)^{\tau k}\left(1+2 \delta_{2}\right)^{\tau k}} \frac{\left|\bar{c}_{0}\right|+\left|\bar{d}_{0}\right|}{2}\left(1-2 \delta_{2}\right)^{k} .
$$

The r.h.s. of (26) tends to zero as $k \rightarrow \infty$ if

$$
\tau \geq \tau_{2}=\frac{\ln \left(1-2 \delta_{2}\right)}{\ln \left[\left(1+\mu \delta_{1}\right)\left(1+2 \delta_{2}\right)\right]-\ln \mu}, \quad \tau_{2}<0 .
$$

Therefore, $\tau^{*}\left(m_{0}\right) \in\left[\tau_{2}, \tau_{1}\right]$, so vector bundle $E$ is $C^{r^{\prime}}$ smooth, where $r^{\prime}<1 / \tau_{1}, r^{\prime} \leq r-2$.

Existence of stable manifold $W^{s}(\gamma)$ and its smoothness can be proved in a similar way. 


\section{REFERENCES}

[1] Arnold V. I., Mathematical methods of classical mechanics. New York: Springer-Verlag, 1989. MR997295(90c:58046)

[2] Arnold V., Kozlov V., Neishtadt A., Mathematical aspects of classical and celestial mechanics, Dynamical systems III. Berlin: Springer-Verlag, 2006. MR.2269239(2008a:70001)

[3] Arnold V. I., Givental A. B., Symplectic Geometry and its Applications, Dynamical Systems IV, Eds. Arnold V. I. \& Novikov S. P. New York: Springer-Verlag, 1990. MR1042758 (90j:58039)

[4] Bolotin S. V., Homoclinic orbits to invariant tori of Hamiltonian systems, AMS Transl. Ser. 2. 1995. Vol. 168. P. 21-90. MR 1351032 (97b:58124)

[5] Bolotin S. V., Treschev D. V., Remarks on the definition of hyperbolic tori of Hamiltonian systems, Regul. Chaotic Dyn. 2000. Vol. 5, No. 4. P. 401-412. MR.1810623(2002h:37119)

[6] Celletti A., Negrini P., Non-integrability of the problem of motion around an oblate planet, Cel. Mech. Dynam. Astron. 1995. Vol. 61. P. 253-260. MR.1335526 (96f:70018)

[7] Conley C. C., On the ultimate behavior of orbits with respect to an unstable critical point. I. Oscillating, asymptotic, and capture orbits, J. Diff. Equat. 1969. Vol. 5. P. 136-158. MR0251301 (40:4532)

[8] Cresson J., Symbolic dynamics and Arnold diffusion, J. Diff. Equat. 2003. Vol. 187. P. $269-292$. MR.1949441 (2004d:37088)

[9] Cushman R., Examples of nonintegrable analytic Hamiltonian vector fields with no small divisors, Trans. AMS. 1978. Vol. 238, No. 1. P. 45-55. MR0478223 (57:17709)

[10] Delshams A., Gutiérrez P., Splitting potential and the Poincaré-Melnikov method for whiskered tori in Hamiltonian systems, J. Nonlinear Sci. 2000. Vol. 10, No. 4. P. 433-476. MR.1766491 (2001d:37094)

[11] Delshams A., de la Llave R., Seara T. M., Geometric properties of the scattering map of a normally hyperbolic invariant manifold, Adv. Math. 2008. Vol. 217, No. 3. P. 1096-1153. MR2383896 (2008m:37100)

[12] Devaney R. L., Homoclinic orbits in Hamiltonian systems, J. Diff. Equat. 1976. Vol. 21, No. 2. P. 431-438. MR 0442990 (56:1365)

[13] Easton R., Homoclinic phenomena in Hamiltonian systems with several degrees of freedom, J. Diff. Equat. 1978. Vol. 29. P. 241-252. MR502350(80d:58026)

[14] Fenichel N., Asymptotic stability with rate conditions, Indiana Univ. Math. J. 1974. Vol. 23, No. 12. P. 1109-1137. MR0339276 (49:4036)

[15] Fenichel N., Asymptotic stability with rate conditions, II, Indiana Univ. Math. J. 1977. Vol. 26, No. 1. P. 81-93. MR0426056 (54:14002)

[16] Franks J., Rotation numbers and instability sets, Bull. AMS. 2003. Vol. 40, No. 3. P. $263-279$. MR.1978565(2004h:37063)

[17] Gonchenko S. V., Shilnikov L. P., Turaev D. V., Elliptic periodic orbits near a homoclinic tangency in four-dimensional symplectic maps and Hamiltonian systems with three degrees of freedom, Reg. Chaot. Dyn. 1998. Vol. 3, No. 4. P. 3-26. MR1704979(2000h:37084)

[18] Grotta Ragazzo C., Nonintegrability of some Hamiltonian systems, scattering and analytic continuation, Comm. Math. Phys. 1994. Vol. 166. P. 255-277. MR1309550 (95m:58058)

[19] Grotta Ragazzo C., Irregular dynamics and homoclinic orbits to Hamiltonian saddle centers, Comm. Pure Appl. Math. 1997. Vol. 50, No. 2. P. 105-147. MR.1426709 (98d:58151)

[20] Grotta Ragazzo C., Koiller J., Oliva W. M., On the motion of two-dimensional vortices with mass, J. Nonlinear Sci. 1994. Vol. 4, No. 5. P. 375-418. MR1291115 (95e:58066)

[21] Hirsch M. W., Pugh C. C., Shub M., Invariant manifolds. Berlin-New York: Springer-Verlag, 1977. (Lecture Notes in Math.; Vol. 583). MR0501173 (58:18595)

[22] Kelley A., The stable, center-stable, center, center-unstable, unstable manifolds, J. Diff. Equat. 1967. Vol. 3. P. 546-570. MR0221044(36:4096)

[23] Koltsova O. Yu., Lerman L. M., Periodic and homoclinic orbits in a two-parameter unfolding of a Hamiltonian system with a homoclinic orbit to a saddle-center, Int. J. Bifurcation \& Chaos. 1995. Vol. 5, No. 2. P. 397-408. MR.1341274 (96f:58060)

[24] Koltsova O., Lerman L., Families of transverse Poincaré homoclinic orbits in 2N-dimensional Hamiltonian systems close to the system with a loop to a saddle-center, Int. J. Bifurcation \& Chaos. 1996. Vol. 6, No. 6. P. 991-1006. MR.1409407 (97g:58060)

[25] Koltsova O., Lerman L., Delshams A., Gutiérrez P., Homoclinic orbits to invariant tori near a homoclinic orbit to center-center-saddle equilibrium, Phys. D. 2005. Vol. 201, No. 3-4. P. 268-290. MR 2123447(2005m:37140)

[26] Kozlov V. V., Integrability and non-integrability in Hamiltonian mechanics, Russ. Math. Surv. 1983. Vol. 38. P. 1-76. MR693718(84k:58076) 
[27] de la Llave R., Some recent progress in geometric methods in the instability problem in Hamiltonian mechanics, Intern. Congr. of Mathematicians (Madrid, Spain, 2006). Vol. 2. Zürich: Eur. Math. Soc., 2006. P. 1705-1729. MR2275666 (2007m:37133)

[28] Lerman L. M., Complex dynamics and bifurcations in Hamiltonian system having the transversal homoclinic orbit to a saddle focus, Chaos. 1991. Vol. 1, No. 2. P. 174-180. MR1135905(92h:58152)

[29] Lerman L. M., Umanskiy Ya. L., On the existence of separatrix loops in four-dimensional systems similar to the integrable Hamiltonian systems, Prikl. Mat. Mekh. 1983. Vol. 47, No. 3. P. 395-401 (in Russian). (Engl. transl. J. Appl. Math. Mech., 1983, vol. 47, No. 3, p. 335-340). MR760166 (86b:58043)

[30] Lerman L. M., Hamiltonian systems with a separatrix loop of a saddle-center, Methods of Qualitative Theory of Diff. Equat. / Ed. E. A. Leontovich-Andronova. Gorky State Univ., 1987. P. 89-103 (Russian) (Engl. transl. Selecta Math. Sov., 1991, vol. 10, No. 3, p. 297-306). MR1120110

[31] Lidov M. L., Vashkov'yak M. A., Doubly asymptotic symmetric orbits in the plane restricted circular three-body problem. Preprint No. 115, Inst. for Applied Math. of USSR Acad. of Sci., Moscow, 1975 (in Russian).

[32] Llibre J., Martínez R., Simó C., Tranversality of the invariant manifolds associated to the Lyapunov family of periodic orbits near $L_{2}$ in the restricted three-body problem, J. Diff. Equat. 1985. Vol. 58, No. 1. P. 104-156. MR791842(87a:58066)

[33] Melnikov V. K., On the stability of a center for time-periodic perturbations, Trudy Moskov. Mat. Obsch. 1963. Vol. 12. P. 3-52 (in Russian). MR0156048 (27:5981)

[34] Mielke A., Hamiltonian and Lagrangian flows on center manifolds. Berlin: Springer-Verlag, 1991. (Lecture Notes in Math.; Vol. 1489). MR.1165943 (93f:58080)

[35] Mielke A., Holmes P., O'Reilly O., Cascades of homoclinic orbits to, and chaos near, a Hamiltonian saddle-center, J. Dyn. Diff. Equat. 1992. Vol. 4, No. 1. P. 95-126. MR.1150399 (93c:58161)

[36] Moser J., Lectures on Hamiltonian systems, Memoirs of AMS. 1968. Vol. 81. MR0230498 (37:6060)

[37] Robinson C., Horseshoes for autonomous Hamiltonian systems using the Melnikov integral, Ergod. Theory Dyn. Syst. 1988. Vol. 8. P. 395-409. MR.967646 (89m:58100)

[38] Rüssmann H., Kleine Nenner I: Über invarianten Kurven differenzierbarer Abbildungen eines Kreisringes, Nachr. Acad. Wiss. Göttingen Math. Phys. Kl. II. 1970. P. 67-105. MR0273156 (42:8037)

[39] Smale S., Differential dynamical systems, Bull. AMS. 1967. Vol. 73. P. 747-817. MR0228014 $(37: 3598)$

[40] Turaev D. V., Shilnikov L. P., On Hamiltonian systems with homoclinic saddle curves, Soviet Math. Dokl. 1989. Vol. 39. P. 165-168. MR988994(90d:58047)

[41] Treshchev D., Hyperbolic tori and asymptotic surfaces in Hamiltonian systems, Russian J. Math. Phys. 1994. Vol. 2, No. 1. P. 93-110. MR.1297943 (95g:58204)

[42] Zakharov V. E., Manakov S. V., Novikov S. P., Pitaevsky L. P., Theory of solitons. The inverse problem method. Plenum Press, 1999.

Department of Differential Equations \& Mathematical Analysis - and - Research InStitute for Applied Mathematics \& Cybernetics, Lobachevsky State University of Nizhny NOVGOROD

E-mail: lermanl@mm.unn.ru

Department of Differential Equations \& Mathematical Analysis - and - Research Institute for Applied Mathematics \& Cybernetics, Lobachevsky State University of Nizhny NOVGOROD

E-mail: anijam@yandex.ru 